\title{
Low oxygen enhances trophoblast column growth by potentiating the extravillous lineage and promoting LOX activity
}

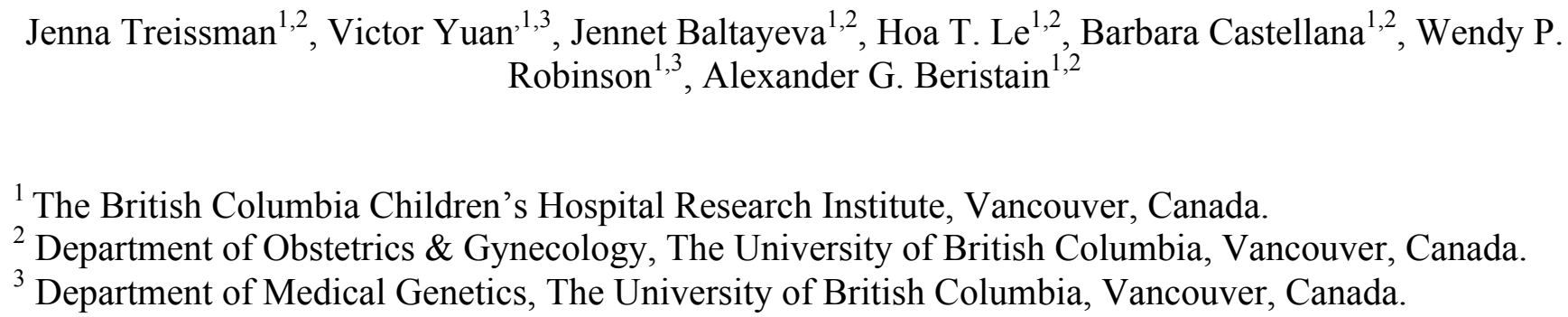

To whom correspondence should be addressed: Alexander G. Beristain, The British Columbia 14 Children's Hospital Research Institute, The University of British Columbia, Vancouver, British

Keywords: Hypoxia, placentation, trophoblast, extravillous trophoblast, villous cytotrophoblast, lysyl oxidase

Summary Statement: Low oxygen promotes extravillous trophoblast differentiation 


\section{Abbreviations}

24 BAPN: $\beta$-aminopropionitrile

25 BrdU: Bromodeoxyuridine

26 CM: Conditioned media

27 CTB: Cytotrophoblast

28 DAP: 1,5-diaminopentane

29 DCT: Distal column trophoblast

30 DGE: Differential gene expression

31 ECM: Extracellular matrix

32 EVT: Extravillous trophoblast

33 FDR: False discovery rate

34 GO: Gene ontology

35 HIF1A: Hypoxia inducible factor $1 \mathrm{~A}$

36 HLA-G: Human leukocyte antigen G

37 Hr: Hour

38 IF: Immunofluorescence

39 LOX: Lysyl oxidase

40 PCA: Principal component analysis

41 PCT: Proximal column trophoblast

42 PEG10: Paternally expressed gene 10

43 scRNA-seq: single cell RNA sequencing

44 SCT: Syncytiotrophoblast

45 UMAP: Uniform manifold approximation and projection

46 vCTB: Villous cytotrophoblast 


\section{ABSTRACT}

50 Early placental development and the establishment of the invasive trophoblast lineage take place within

51 a low oxygen environment. However, conflicting and inconsistent findings have obscured the role of

52 oxygen in regulating invasive trophoblast differentiation. In this study, the effect of hypoxic, normoxic,

53 and atmospheric oxygen on invasive extravillous pathway progression was examined using a human

54 placental explant model. Here, we show that exposure to low oxygen enhances extravillous column

55 outgrowth and promotes the expression of genes that align with extravillous trophoblast (EVT) lineage

56 commitment. By contrast, super-physiological atmospheric levels of oxygen promote trophoblast

57 proliferation while simultaneously stalling EVT progression. Low oxygen-induced EVT differentiation

58 coincided with elevated transcriptomic levels of lysyl oxidase $(L O X)$ in trophoblast anchoring columns,

59 where functional experiments established a role for LOX activity in promoting EVT column outgrowth.

60 The findings of this work support a role for low oxygen in potentiating the differentiation of

61 trophoblasts along the extravillous pathway. Additionally, these findings generate insight into new

62 molecular processes controlled by oxygen during early placental development. 


\section{INTRODUCTION}

In mammalian development, the placenta forms the mechanical and physiological link between

67 maternal and fetal circulations. In rodents and humans that have invasive haemochorial placentae, nutrient and oxygen transfer between mother and fetus is achieved through extensive uterine infiltration by placenta-derived cells of epithelial lineage called trophoblasts (Pijnenborg et al., 2011; Velicky et al., 2016). In humans, trophoblast differentiation into invasive cell subtypes, called extravillous trophoblast (EVT), is essential for optimal placental function (Tilburgs et al., 2015; Velicky et al., 2016). Molecular processes governing invasive EVT differentiation and specific EVT functions like uterine artery remodeling and immuno-modulation of maternal leukocytes are strictly controlled (Pollheimer et al., 2018; Wallace et al., 2012). Defects in trophoblast differentiation along the EVT pathway associate with impaired placental function and certain aberrant conditions of pregnancy that directly impact fetal and maternal health (Avagliano et al., 2012).

In early pregnancy, anchoring villi of the placental basal plate initiate cellular differentiation events leading to the formation of EVT. At specific villi-uterine attachment points, proliferative EVT progenitors establish multi-layered cellular structures called anchoring columns (Haider et al., 2016; Pollheimer et al., 2018). Trophoblasts residing within proximal regions of anchoring columns, termed proximal column trophoblasts (PCT), are highly proliferative and show evidence of initial molecular characteristics that are hallmarks of EVT (Haider et al., 2018; Turco et al., 2018). At distal regions within anchoring columns, column trophoblasts lose their proliferative phenotype and express many markers akin to invasive EVT, such as HLA-G, $\alpha 5$ and $\beta 1$ integrins, NOTCH2, and ERBB2 (Fock et al., 2015a; Fock et al., 2015b; Haider et al., 2016; Kabir-Salmani et al., 2004; Zhou et al., 1997). The transition of PCT into distal column trophoblasts (DCT) represents a significant developmental step towards the formation of uterine-invading and tissue-remodeling EVT.

Anchoring columns of the placenta initially develop in the absence of maternal blood, and 
subsequently, within a relatively low oxygen environment $(\sim 20 \mathrm{mmHg})$ (Jauniaux et al., 2000; Jauniaux et al., 1999; Rodesch et al., 1992). By comparison, the partial pressure of arterial blood oxygen is approximately $100 \mathrm{mmHg}$, while the partial pressure of oxygen within the placental bed by

14 weeks' gestation is estimated to be between 40-60 mmHg (Jauniaux et al., 2001). The role of oxygen in controlling anchoring column formation and EVT differentiation has been the focus of many studies (Caniggia et al., 2000; Genbacev et al., 1997; James et al., 2006; Lash et al., 2006; Wakeland et al., 2017). Unfortunately, contradictory and inconsistent findings have obscured the role of oxygen tension in controlling aspects of trophoblast biology, where low oxygen both promotes (Caniggia et al., 2000; Genbacev et al., 1997) and restrains (James et al., 2006; Lash et al., 2006) column outgrowth, and potentiates (Wakeland et al., 2017) and inhibits (Caniggia et al., 2000; Genbacev et al., 1997) EVT differentiation. These reported differences on the effect of oxygen on anchoring column outgrowth and EVT differentiation are likely attributed to differences in model platforms and methods used to isolate, characterize, and culture trophoblasts. Nonetheless, the role of oxygen in controlling anchoring column formation and column trophoblast differentiation has yet to be fully examined. outgrowth. Using placental villous tissue explants that recapitulate many of the morphological and molecular events central to anchoring column formation and EVT differentiation in vivo, we show that low levels of oxygen potentiate column outgrowth. We demonstrate that low oxygen drives hypoxiarelated gene programs and processes central to cell-extracellular matrix interaction, while exposure to high oxygen promotes/maintains a strong proliferative phenotype. Moreover, we provide evidence that supports a role for low levels of oxygen in promoting the differentiation of column trophoblasts along the EVT pathway. We further identify $L O X$ as a critical gene up-regulated in response to low oxygen that supports column outgrowth, and provide important insight into novel molecular programs impacted by oxygen that align with trophoblast differentiation along the EVT pathway. 


\section{RESULTS}

\section{Hypoxia potentiates trophoblast column outgrowth}

To test the effect of exposure to differing levels of oxygen on trophoblast column establishment

117 and outgrowth, we utilized a Matrigel-imbedded placental explant model that closely reproduces 118 developmental processes of trophoblast column cell expansion and differentiation along the EVT 119 pathway (Bilban et al., 2009; Newby et al., 2005). Early column formation during human placental 120 development is characterized by the expansion of mitotically active $\mathrm{Ki}^{+} 7^{+} / \mathrm{HLA}-\mathrm{G}^{\mathrm{lo}}$ proximal column 121 trophoblasts (PCT) into HLA-G ${ }^{\text {hi }}$ non-proliferating distal column trophoblasts (DCT) and invasive

122 EVT (Figure 1A, 1B). To this end, Matrigel-imbedded placental explants recapitulate anchoring 123 column cell organization and EVT-lineage commitment, establishing the explant system as an 124 appropriate experimental tool to study the cellular and molecular underpinnings regulating human 125 trophoblast column formation and outgrowth (Figure 1C). imbedded into Matrigel matrix and allowed to establish for $24 \mathrm{hr}$ at 5\% oxygen (Figure 2A); this level of oxygen represents a relative "normoxic" condition for early placental development (Jauniaux et al., 2001). Characteristics (age, gestational age, BMI, smoking status) of each patient who donated their placenta for explant culture are listed in Supplemental Table 1. Following this, explants derived from

131 the same placenta were transferred into one of three conditions for an additional $48 \mathrm{hr}$ representing 132 either hypoxic $\left(1 \%, \sim 10 \mathrm{mmHg} \mathrm{O}_{2}\right)$, normoxic $\left(5 \%, \sim 35 \mathrm{mmHg} \mathrm{O}_{2}\right)$, or hyperoxic $(20 \%, \sim 141 \mathrm{mmHg}$ $133 \mathrm{O}_{2}$ ) environments relative to the first trimester of pregnancy (Figure 2A). Within all oxygen conditions, 134 explant outgrowth was observed (Figure 2B, 2C). However, column outgrowth was most pronounced 135 in $1 \%$ and $5 \%$ oxygen, where outgrowth area in both of these low oxygen conditions was significantly 136 greater than outgrowth observed in $20 \%$ oxygen (Figure 2C, 2D). While column outgrowth in $5 \%$ 137 oxygen was overall less variable and trended on producing smaller columns than explants cultured in 
$1381 \%$ oxygen, there was no statistical difference between outgrowth in $1 \%$ and $5 \%$ oxygen (Figure 2D).

139 In summary, trophoblast column outgrowth was potentiated by low oxygen, whereas exposure to 140 atmospheric oxygen (20\% oxygen) blunted column outgrowth.

142 Explant exposure to low or high levels of oxygen generate distinct transcriptomic signatures

144 oxygen modulate trophoblast column outgrowth, global gene expression was analysed in placental 145 explant cultures using gene microarrays. For this experiment, placental explants from 5 unique 146 placentae ( $\mathrm{n}=5 ; 5-7$ weeks' gestation) were established as previously described except that cultures 147 were maintained in their respective oxygen condition $(1 \%, 5 \%$, or $20 \%)$ for 24 hr prior to RNA 148 isolation in order to capture molecular signatures central to column formation. Importantly, RNA was 149 extracted from only column trophoblasts and Matrigel-invading EVT; chorionic villi of explants were carefully micro-dissected away from columns and EVT following an approach described within Bilban et al (Bilban et al., 2009) (Figure 3A). Following standard probe filtering, and normalization 152 (Supplemental Figure 1), differential gene expression (DGE) analysis of explant trophoblasts was 153 performed. Using a false discover rate $(\mathrm{FDR})<0.05$, we identified many differences in gene 154 expression between 1\% versus 20\% (293 genes up-regulated; 685 genes down-regulated) and 5\% versus 20\% oxygen conditions (363 genes up-regulated; 406 genes down-regulated) (Figure 3B;

156 Supplemental Table 2). DGE analysis between $1 \%$ and $5 \%$ oxygen did not identify differentially 157 expressed genes (Figure 3B). Principal component analysis (PCA) shows clustering of explants 158 cultured in hypoxic, normoxic, and hyperoxic conditions (Figure 3C). PCA sample clustering showed 159 explant trophoblasts cultured in $1 \%$ and $5 \%$ oxygen generally clustered closer together, save for two $1601 \%$ oxygen explant outliers; one clustered amongst $20 \%$ oxygen samples while the other clustered 161 separately to all other samples (Figure 3C). Despite these two explants being identified as significant 
outliers using the Silhouette coefficient (Barghash and Arslan, 2016), we opted to retain them for the

163 remainder of our analyses as we could not confidently ascribe outlier classification due to technical or 164 batch-related artifacts.

Hierarchical clustering of the 15 column trophoblast samples segregated samples into two statistically significant clusters (sigclust, $\mathrm{p}<0.05$ ): a 20\% oxygen dominated cluster and a cluster comprised of column trophoblasts cultured in $1 \%$ and $5 \%$ oxygen (Figure 3D). A gene heat-map of the top 40 differentially expressed genes (top 20 differentially expressed genes in 1\%; top 20 differentially expressed genes in $20 \%$; FDR $<0.05$, ranked by FC) highlights gene patterns across $1 \%, 5 \%$, and $20 \%$ oxygen cultures (Figure 3D). In both $1 \%$ and $5 \%$ oxygen conditions, the top hits identified by global DGE analysis included genes associated with hypoxia (EGLN3, RORA), cell-matrix interaction/restructuring ( $L O X, J A M 2, E G L N 3, P L A U R)$, and gene transcription regulation (MXI1, TSC22D3, 173 RORA). In contrast, the most highly expressed genes in explants exposed to $20 \%$ oxygen were exclusive to pro-mitotic/proliferative processes (MKI67, KIF20A, KIF23, PEG10, CDK1, NCAPG, $N C A P H, T O P 2 A, C D C 7)$, indicating that column trophoblasts cultured in $20 \%$ oxygen possess a proliferative phenotype. and 5\% oxygen culture conditions, we did identify 123 and 53 unique genes to be up-regulated in 5\% and $1 \%$ oxygen cultures compared to samples cultured in $20 \%$ oxygen (Figure 3E). Further, 78 and 357 genes were shown to be uniquely down-regulated in 5\% and 1\% oxygen cultured explants compared to $20 \%$ cultures (Figure 3F). Gene ontology (GO) pathway analysis of the above 1\% and 5\% signatures indicated that column trophoblasts exposed to $1 \%$ oxygen show enrichment for processes favoring 183 hypoxia-related- and oxidative stress-signaling (Supplemental Figure 2). Conversely, column 184 trophoblasts cultured in 5\% oxygen showed enrichment of biological pathways linked to nucleotide 185 biosynthesis and metabolism, indicating that column trophoblasts in $1 \%$ and $5 \%$ oxygen do exhibit 
underlying molecular differences potentially contributing to trophoblast column development

187 (Supplemental Figure 2).

\section{Differing oxygen levels drive distinct molecular programs in trophoblast columns}

To broadly examine how transcriptomic differences within column trophoblasts exposed to

hypoxic, normoxic, and hyperoxic conditions relate to differences in molecular pathways, gene signatures determined by DGE analysis (FDR < 0.05; fold-change $>1.5$; Supplemental Table 2) were used to identify pathways enriched in explant column trophoblasts cultured in $1 \%, 5 \%$, and $20 \%$ oxygen. Unsurprisingly, pathways in explants cultured in 1\% oxygen (293 genes; clusterProfiler) showed enrichment for multiple pathways and molecular processes specific to hypoxia (Figure 4A). Additionally, the $1 \%$ oxygen signature also showed enrichment for pathways related to extracellular matrix (ECM) structure and organization, steroid hormone responses, and hydroxyproline metabolism

enrichment for genes specific to ECM composition, response to hypoxia, and response to steroid hormones, but also showed enrichment of pathways related to bone development and viral entry into cells (Supplemental Figure 3). By contrast, 20\% oxygen (685 genes) showed enrichment of pathways and cellular processes related to organelle fission, nuclear division, chromosome segregation, mitotic nuclear division, and DNA packaging and replication, all of which link to heightened cell cycle activity and proliferation (Figure 4B).

GO gene signatures enriched within 20\% column trophoblasts (93 genes; Supplemental Table 3), explant samples were subjected to hierarchical clustering and visualized by gene heat-map (Figure 4C). 
$2105 \% / 20 \%$ samples) (Figure 4C). This later branch was further divided into two sub-branches, one

211 enriched by 5\% oxygen samples also containing a 1\% oxygen sample outlier, and the other sub-branch

212 made up entirely of $20 \%$ oxygen column trophoblasts (Figure 4C). Interestingly, gene heat-map

213 expression intensities suggest a step-wise increase in expression of pro-mitotic/proliferative genes in

214 column trophoblasts exposed to increasing levels of oxygen (Figure 4C). To verify if an increase in

215 exposure to oxygen tension translates into increased proliferation, a BrdU pulse-chase was performed

216 on a separate cohort of placental explants $(n=3)$ cultured in $1 \%, 5 \%$, and $20 \%$ oxygen. In support of the

217 gene array data, little/no evidence of cell proliferation within explant columns was observed in $1 \%$

218 oxygen cultures following a $4 \mathrm{hr}$ chase (Figure 4D, 4E). However, explants cultured in 5\% oxygen

219 showed a significant increase in BrdU incorporation within column trophoblasts compared to $1 \%$

220 oxygen columns, and an even greater level of BrdU positivity was measured within $20 \%$ oxygen

221 columns $(20 \%$ versus $1 \%)$ (Figure 4D, 4E). Though a trend for greater proliferation in explant columns

222 cultured in $20 \%$ oxygen compared to $5 \%$ oxygen was observed, this difference was not significant

223 (Figure 4E). Overall, our findings suggest that explant column trophoblasts cultured in low oxygen up-

224 regulate molecular processes related to hypoxia/HIF1A signalling and ECM organization/remodeling,

225 while column trophoblasts exposed to hyper-physiological $20 \%$ oxygen adopt a predominantly pro-

226 proliferative phenotype.

\section{Low oxygen promotes EVT differentiation}

Differentiation of trophoblasts along the EVT pathway is in part defined by the exiting of PCT

located at the base of anchoring columns from the cell cycle (Velicky et al., 2018). Moreover, as

231 column trophoblasts located at distal portions of columns acquire pro-invasive EVT-like

232 characteristics, molecular pathways related to ECM-remodeling and protease functions are accordingly

233 up-regulated (Davies et al., 2016). Our observation that low oxygen (1\% \& 5\%) promotes 
234 transcriptional signatures linked to cell-ECM interaction and protease-ECM remodeling, while $20 \%$ 235 oxygen promotes proliferation, suggests that exposure to low oxygen drives, while high oxygen 236 restrains EVT differentiation.

To gain insight into how differing levels of oxygen affect column trophoblast differentiation, explant column trophoblasts were subjected to hierarchical cluster analysis using a signature of differentially expressed genes derived from a list of 47 trophoblast-related genes curated from recent high-dimensional data and differentiation studies focused on trophoblast biology (Supplemental Figure

Turco et al., 2018). This list includes genes associated with trophoblast lineage (TFAP2A, KLF5, 243 GATA3), trophoblast pluripotency (CDX2), villous cytotrophoblast (CTB) state (EGFR, SPINT1, 244 ITGA6, PEG10, TEAD4, TP63), EVT state (HLA-G, HTRA1, LAIR2, FLT-1, ERBB2, ADAM12, 245 AMAM19, MYC, ITGA5, TEAD2), syncytiotrophoblast (SCT) state (GCM1, CGA, ERVW1, ERVFRD-1, 246 ENDOU), and genes commonly used to identify proliferative CTB and PCT (MK167, CCNA2, 247 NOTCH1). From this curated list, 14 genes were differentially expressed between 1\%, 5\%, and 20\% 248 oxygen cultured explants (Supplemental Figure 4A). Notably, this small signature was sufficient to 249 segregate samples into two main groups: One group consisted almost entirely of column trophoblasts 250 cultured in $20 \%$ oxygen (save for one $1 \%$ oxygen outlier), while the other group was made up of a mix 251 of $1 \%$ and 5\% oxygen cultured samples (Supplemental Figure 4A). Genes enriched within low-oxygen 252 cultured explants included trophoblast lineage-related transcription factors (GATA3, KLF5, TFAP2A), 253 genes related to ECM remodeling (TIMP1, ADAM12, ADAM19), genes related to CTB specification $254(C D H 1, E G F R)$, and genes linked with the EVT sub-lineage (ADAM12, FLT1, ITGA5, MYC) 255 (Supplemental Figure 4A). Samples that grouped primarily as 20\% oxygen cultured explants showed 256 enrichment for genes specific to proliferative CTB and PCT (CCNA2, MKI67). Notably, the imprinted 257 paternally expressed gene, PEG10, was also highly expressed within $20 \%$ oxygen column trophoblasts 
258 (Supplemental Figure 4A).

Examination of PEG10 localization within first trimester placental villi ( $\mathrm{n}=3$ placentae; 6-10 weeks' gestation) by immunofluorescence microscopy (IF) showed that PEG10 preferentially localizes (established from $n=5$ placentae) revealed that PEG10 signal, similar to the signal observed in placental villi, is broadly localized to CTB in explants exposed to all three oxygen culture conditions (Supplemental Figure 4C). However, within 20\% oxygen explants, bright PEG10 signal was also observed within multi-layered PCT; this column-specific signal was minimal/absent in 1\% and 5\% oxygen cultures (Supplemental Figure 4C).

To more closely examine how global gene expression changes identified within low and high oxygen exposed explants relate to trophoblast differentiation, we examined the expression of the top fifteen up-regulated genes in column trophoblasts cultured in $1 \%$ and $20 \%$ oxygen (FDR $<0.05$; foldchange $>2$ ) within a recently reported first trimester placenta single cell transcriptomic dataset (VentoTormo et al., 2018). Using this dataset we focused exclusively on the 5 subtypes of trophoblasts that were described (14,366 trophoblasts from 5 individual placentae): CTB, proliferative CTB, SCT, proliferative EVT (likely PCT), and EVT (likely an admixture of DCT and invasive EVT) (Figure 5A) (Vento-Tormo et al., 2018). Within UMAP-directed cell clusters, the specificity of each trophoblast sub-lineage/type is shown by expression levels of EGFR (CTB), ERVFRD-1 (syncytin-2; SCT), HLA-G 276 (column trophoblast \& EVT), and MKI67 (proliferating trophoblast) (Figure 5B). A heat-map projection shows the pattern of gene expression of trophoblast lineage and subtype-specific genes (Pan trophoblast: KRT7, TFAP2A, GATA3, KLF5; CTB: EGFR, SPINT1, TP63; proliferative CTB and PCT: MKI67, CCNA2; SCT: ENDOU, ERVFRD-1; EVT: HLA-G, ITGA5, ERBB2), and the fifteen top upregulated genes in 20\% (OAS1, IFIT3, DLGAP5, GSTA3, CDK1, OXCT1, CENPF, KIF20A, PARP1, 
AKAP12, ACTC1, BIRC7, SPNS2, S100A4, JAM2, MXI1, EGLN3, RORA, PLAUR, AK4, TSC22D3,

NRN1) (Figure 5C). Interestingly, genes up-regulated within 1\% oxygen-cultured columns showed 284 alignment with proliferative EVT, and this relationship was even greater with EVT (Figure 5C). In contrast, the top genes identified within $20 \%$ oxygen columns aligned predominately with proliferative CTB and proliferative EVT (Figure 5C). reproduced a lineage trajectory similar to that reported in Vento-Tormo et al (Vento-Tormo et al., 2018), where a predicted cell origin state was identified as well as the two differentiation trajectories aligning with the villous and extravillous pathways (Figure 5D). Correlating the expression of genes specific to distinct states of trophoblast differentiation (i.e. EGFR, HLA-G, ERVFRD-1) with the pseudotime trajectory confirmed the accuracy of the pseudotime modeling, where EGFR aligned to cells committed to the villous pathway, $H L A-G$ to a maturing EVT, and $E R V F R D-1$ to a terminally differentiated SCT (Figure 5E). Correlating trophoblast lineage trajectory with the top up-regulated $1 \%$ and $20 \%$ oxygen genes showed that genes enriched within $20 \%$ oxygen aligned to both the villous and extravillous pathway trajectories (Figure 5F). Notably, high-oxygen genes aligned closely with cell states linked to proliferative CTB (PARP1, RFX5, PLEKHH1, GSTA3, NRP2) and proliferative PCT (OXCT1, KIF20A, NCAPH, CENPF, TOP2A, DLGAP5, CDK1, KPNA2) (Figure 5F). By contrast, low oxygen enriched genes primarily aligned with the tail-end of the extravillous pathway (i.e. TNFSF10, LOX, SPNS2, S100A4, JAM2, PLAUR) (Figure 5F). Together, these data suggest that column trophoblasts exposed to low oxygen adopt transcriptomic signatures that are reflective of EVT, while column trophoblasts cultured in $20 \%$ oxygen express genes that align predominately with proliferating 


\section{$L O X$ expression and activity is potentiated by low oxygen}

Our finding that low oxygen drives column outgrowth and potentiates the expression of genes

linked with the EVT phenotype suggests that genes highly expressed within low oxygen columns may

in part contribute to EVT differentiation and trophoblast column formation. Rank ordering of up-

regulated genes by fold-change in both $1 \%$ and 5\% oxygen column trophoblasts identified multiple

encoding lysyl oxidase, a copper-dependent enzyme that catalyses collagen and elastin crosslinking,

was the number 2-ranked gene in both $1 \%$ and $5 \%$ oxygen cultured explants. Specifically, $L O X$

expression was 6.2- and 5.1-fold higher in $1 \%$ and $5 \%$ oxygen cultures compared to $20 \%$ oxygen

explants (Figure 6A; Supplemental Table 2). While previous work has identified a role for elevated

of LOX in placental trophoblast column biology and trophoblast differentiation along the EVT pathway has not been described.

As an initial step to examine the importance of LOX in anchoring column biology, LOX mRNA in situ hybridization within first trimester placental villi $(\mathrm{n}=3 ; 6-8$ weeks' gestation) was performed.

RNAscope in situ hybridization showed specific and intense $L O X$ localization to cells within the mesenchymal core of placental villi and to trophoblasts within anchoring columns (Figure 6B). placental explants cultured in $1 \%$ and $20 \%$ oxygen supported the gene array finding that $L O X$ expression was elevated in column trophoblasts exposed to low oxygen (Figure 6C). While we were unable to verify elevated LOX protein expression in low oxygen-cultured placental explants via IF microscopy due to non-specific antibody signal, LOX enzymatic activity, measured in conditioned media (CM) generated by placental explants cultured in $1 \%$ or $20 \%$ oxygen, showed that activity was 
330 (BAPN), demonstrated LOX-substrate specificity, while recombinant active LOX served as a positive 331 control (Figure 6D). Taken together, these findings show that LOX expression and activity is elevated 332 in column trophoblasts cultured in low oxygen. Further, LOX's preferential in vivo expression within 333 the trophoblast anchoring column combined with its involvement in promoting tumor cell metastasis, 334 suggests that LOX may also play a role in controlling trophoblast column outgrowth and/or EVT 335 differentiation.

\section{Impairment of LOX restrains column outgrowth}

To test the function of LOX in column outgrowth, Matrigel-imbedded placental explants cultured in $1 \%$ oxygen $(n=3)$ were cultured in either control explant media or media containing the competitive LOX inhibitor BAPN. The effectiveness of BAPN in inhibiting LOX activity was measured as before by examining the ability of endogenous LOX in explant CM to oxidize substrate

342 (Figure 7A). Conditioned media harvested from control explants showed LOX activity levels slightly 343 below levels measured in recombinant LOX positive control reactions, but significantly higher than 344 explant media alone (Figure 7A). Importantly, treatment of explants with BAPN significantly blunted 345 LOX activity, though activity was not completely blocked (Figure 7A). Importantly, placental explant 346 treatment with BAPN led to a significant two-fold impairment in column outgrowth (Figure 7B, 7C). 347 Taken together, these results suggest that LOX expression in developing trophoblast columns promotes 348 column outgrowth and associates with an EVT phenotype. 


\section{DISCUSSION}

Here we describe how exposure to different levels of oxygen differentially affect trophoblast column outgrowth and global gene expression. We provide evidence that exposure to low oxygen results in overall increases in column outgrowth accompanied by gene expression signatures that align with an EVT phenotype. By contrast, gene signatures in high oxygen-cultured column trophoblasts define a role for elevated oxygen in maintaining column growth through cell proliferation. In both hypoxic and normoxic conditions, we identify the gene $L O X$, as one of the most highly up-regulated genes within explant columns. We show that $L O X$ expression associates with EVT lineage trajectory and demonstrate that impairment of LOX activity blunts column outgrowth. Together, this work supports a role for low oxygen in potentiating the EVT pathway. Moreover, this work also identifies novel oxygen-sensitive molecular processes that likely play roles in anchoring column formation during human placental development.

The role of oxygen in controlling column formation and the EVT pathway is controversial.

Previous studies have shown that exposure of placental explants to low oxygen (i.e. $2 \%$ to $3 \%$ oxygen) promotes column expansion and outgrowth, where outgrowth is primarily attributed to HIF1A-directed cell proliferation (Caniggia et al., 2000; Genbacev et al., 1997). Consistent with this, evidence exists that CTB exposure to low oxygen restrains trophoblast progression along the EVT pathway (Caniggia et al., 2000; Genbacev et al., 1997; Lash et al., 2006). By contrast, rodents and rodent-derived trophoblast stem cells engineered to lack hypoxia-sensing machinery (i.e. $A R N T, H I F 1 A$, and/or EPSA1 null) fail to differentiate into trophoblast lineages of the labyrinth zone and into trophoblast giant cells, trophoblast populations akin to invasive EVT in humans (Chakraborty et al., 2016; Chakraborty et al., 2011; Cowden Dahl et al., 2005; Gultice et al., 2009; Maltepe et al., 2005). In support of these

372 observations, low oxygen was shown to potentiate an invasive phenotype in human primary 373 trophoblasts with an accompaniment in the expression of EVT-associated genes HLA-G and $\alpha 5$ 
374 integrin, and the up-regulation of pro-migratory integrin-linked kinase signaling (Horii et al., 2016;

375 Robins et al., 2007; Wakeland et al., 2017). Our findings overall align with these later studies that 376 suggest low oxygen promotes differentiation along the EVT pathway. Notably, we show that low 377 oxygen drives expression of EVT-related genes and signatures that align with EVT lineage trajectory. 378 We show that low oxygen (1\% and 5\% oxygen) induces expression of hallmark EVT genes like ITGA5, ADAM12, and FLT1, as well as the transcription factors KLF5 and GATA3 that are 380 preferentially expressed by EVT. Importantly, the use of cell lineage trajectory modeling using single cell RNA sequencing data also provides evidence that low oxygen promotes a cell state consistent with the EVT lineage. These later observations are in line with the association of low levels of oxygen within the intervillous space and anchoring column formation and interstitial EVT infiltration into decidual mucosa in early pregnancy. However, the relationship between relative placental hypoxia and inadequate placentation in aberrant pregnancy conditions like preeclampsia (Farrell et al., 2019) suggests that the impact of oxygen on trophoblast biology may differ according to stage of 387 development.

Our finding that high levels of oxygen promote column trophoblast proliferation is inconsistent with previous studies investigating the role of hypoxia in anchoring column biology (Caniggia et al., 2000; Genbacev et al., 1997). However, in agreement with our findings, James et al reported that explant columns cultured in $8 \%$ oxygen have increased trophoblast cellularity compared with columns exposed to $1.5 \%$ oxygen (James et al., 2006). Moreover, there seems to be consistent agreement that exposure to low oxygen leads to greater column outgrowth, where oxygen tension levels between $1-5 \%$ consistently generate larger columns than atmospheric oxygen conditions (Caniggia et al., 2000; James et al., 2006). However, dissecting how enhanced column outgrowth is achieved, i.e. via greater cell proliferation within the column or through increased trophoblast migration and invasion, is still not completely resolved. Indeed, while our data suggests that low oxygen promotes an EVT phenotype and 
enhances molecular pathways related to cell-ECM remodeling, we did not directly examine if low

oxygen affects EVT invasion. The stark differences on the effect of oxygen levels in regulating trophoblast proliferation and EVT differentiation within explant columns between various studies is difficult to explain, but may stem from differences in media composition, the matrix substratum used in explant cultures, subtle variations in oxygen tension, and the gestational age of placental tissues/cells used for establishing explant cultures. Future studies will need to specifically re-examine how differing levels of oxygen impact column cell proliferation.

PEG10, a paternally expressed and maternally imprinted gene, was identified as a top up-

Peg10 in mouse placental development, where mice deficient in Peg10 show severe fetal growth

suggest that a fine balance in Peg10 expression is required for proper placental development. Notably, in humans, dysregulation of PEG10 associates with certain pregnancy disorders, including pre-

413 eclampsia, gestational hypertension, molar pregnancy, and spontaneous miscarriage (Dória et al., 2010;

414 Liang et al., 2014; Rahat et al., 2017). Our finding that PEG10 expression and cell proliferation in trophoblast columns associates with exposure to high oxygen is consistent with studies showing a role 416 for PEG10 in promoting tumor cell survival and growth (Bang et al., 2015; Ishii et al., 2017; Peng et 417 al., 2017). Recent findings also provide evidence that PEG10 promotes trophoblast cell line 418 proliferation (Abed et al., 2019). Given that PEG10 specifically localizes to CTB of floating villi and to 419 subsets of trophoblasts within proximal regions of anchoring columns suggests that PEG10 may play 420 roles in column establishment by promoting progenitor expansion. Further work is needed to dissect 421 the role of PEG10 in column formation and progression along the EVT pathway. 
Our finding that $L O X$ expression was consistently a gene highly up-regulated in both $1 \%$ and

$4235 \%$ column trophoblasts compared to those cultured in $20 \%$ oxygen indicates that LOX-related

424 processes are important in placental development and trophoblast column biology. While a role of

425 LOX in column formation has not been previously reported, the importance of LOX in tumorigenesis is

426 known, where elevated LOX associates with numerous types of cancers and LOX activity promotes

427 tumor cell metastasis (Cox et al., 2015; Di Stefano et al., 2016). Further, a recent report does provide

428 evidence that LOX expression promotes cell invasion of a trophoblast cell line (Xu et al., 2019). This

429 later finding is consistent with the association of LOX expression and the EVT phenotype. Aldehydes

430 produced by LOX-directed oxidation of lysine residues within collagen and elastin facilitate

431 collagen/elastin cross-linking and stability, which in turn provide a structural lattice for cell movement

432 (Kim et al., 2014). That LOX expression is greatest within both proximal and distal column 433 trophoblasts is interesting, as PCT are not considered to be migratory. Nonetheless, it is likely that 434 creating an appropriate substratum scaffold via LOX-directed collagen crosslinking may contribute to 435 column stability and provide a platform for EVT outgrowth. How or if LOX affects anchoring column 436 establishment or EVT differentiation is unknown, but future studies utilizing newly-derived trophoblast 437 organoids (Haider et al., 2018; Turco et al., 2018) may allow for deep mechanistic examination of LOX 438 and the EVT pathway.

In summary, the extravillous pathway is controlled by multiple intrinsic as well as extrinsic 440 factors, including level of oxygen. We provide evidence that supports a role for low oxygen levels in 441 promoting the differentiation of trophoblasts along the EVT pathway. This finding establishes insight 442 into critical developmental events during placentation that occur in early pregnancy. Further, these 443 findings may also provide a foundation for understanding cellular and molecular processes contributing 444 to conditions linked to aberrant placentation. 


\section{MATERIALS AND METHODS}

\section{Patient recruitment and tissue collection}

448 Decidual and placental tissues were obtained with approval from the Research Ethics Board on the use 449 of human subjects, University of British Columbia (H13-00640). All samples were collected from 450 women (19 to 39 years of age) providing written informed consent undergoing elective terminations of 451 pregnancy at British Columbia's Women's Hospital, Vancouver, Canada. First trimester decidual $452(N=1)$ and placental tissues $(N=30)$ were collected from participating women (gestational ages ranging 453 from 5-12 weeks) having confirmed viable pregnancies by ultrasound-measured fetal heartbeat. The 454 decidual tissue sample was selected based on the presence of a smooth uterine epithelial layer and a 455 textured thick spongy underlayer. Patient clinical characteristics i.e. height and weight were 456 additionally obtained to calculate body mass index (BMI: $\mathrm{kg} / \mathrm{m}^{2}$ ) and all consenting women provided 457 self-reported information via questionnaire to having first hand exposure to cigarette smoke, and are summarized in Supplemental Table 1.

\section{Placental villous explant assay}

Ex vivo placental villous cultures were established as described in (Aghababaei et al., 2014; De

462 Luca et al., 2017; Perdu et al., 2016). Briefly, placental villi from 5-8 week old gestation placentas $463(n=8)$ obtained from patients undergoing elective termination of pregnancy were dissected, washed in 464 cold PBS, and imbedded into Millicell cell culture inserts $(0.4 \mu \mathrm{m}$ pores, $12 \mathrm{~mm}$ diameter. EMD 465 Millipore, Billerica, MA) containing $200 \mu$ of growth-factor-reduced Phenol-red free Matrigel (BD 466 Biosciences, San Diego, CA). Explants, containing $400 \mu \mathrm{l}$ DMEM/F12 1:1 (200 mM L-glutamine) in 467 the outer chamber, were allowed to establish overnight in a humidified $37{ }^{\circ} \mathrm{C}$ trigas incubator at $5 \%$ 468 oxygen, $5 \% \mathrm{CO}_{2}$. Following $24 \mathrm{hr}$ of culture, explants were cultured in $200 \mu \mathrm{DMEM} / \mathrm{F} 12$ 1:1 media 
analyses) or $48 \mathrm{hr}$ (explant outgrowth measurements). All explant media were supplemented with

471 penicillin/streptomycin and antimycotic solution (Thermofisher Scientific, Waltham, MA) Growing 472 explants were imaged at indicated times using a Nikon SMZ 7454T triocular dissecting microscope 473 (Minato, Japan) outfitted with a digital camera. EVT outgrowths were measured by ImageJ software. 474 Fold-change in outgrowth was determined by dividing the mean column area at $48 \mathrm{hr}$ into the mean 475 area at $0 \mathrm{hr}$.

\section{Immunofluorescence, RNAScope, and immunohistochemistry microscopy}

Immunofluorescence: Placental villi (6-12 weeks gestation; $\mathrm{n}=5$ ) or placental explants (derived

from $n=11$ placentae) were fixed in $2 \%$ paraformaldehyde overnight at $4{ }^{\circ} \mathrm{C}$. Tissues were paraffin

embedded and sectioned at $6 \mu \mathrm{m}$ onto glass slides. Immunofluorescence was performed as described

elsewhere (Aghababaei et al., 2015). Briefly, cells or placental tissues underwent antigen retrieval by

heating slides in a microwave for 5 X 2 minute intervals in a citrate buffer $(\mathrm{pH} 6.0)$. Sections were incubated with sodium borohydride for 5 minutes, RT, followed by Triton X-100 permeabilization for

5 minutes, RT. Slides were blocked in 5\% normal goat serum/0.1\% saponin for $1 \mathrm{~h}$, RT, and incubated

with combinations of the indicated antibodies overnight, $4{ }^{\circ} \mathrm{C}$ : Anti-HLA-G $(1: 100,4 \mathrm{H} 84$, Exbio,

Vestec, Czech Republic); anti-cytokeratin 7, mouse monoclonal IgG (1:50, RCK105, Santa Cruz

487 Biotechnology, Dallas, TX); anti-cytokeratin 7, rabbit monoclonal IgG (1:50, SP52, Ventana Medical

Systems, Oro Valley, AZ); anti-Ki67 (1:75, SP6, Thermo Fisher Scientific); anti-LOX (1:100, NB100-

2527, NovusBio, Centennial, CO); anti-PEG10 (1:100, 4C10A7, NovusBio); anti-BrdU (1:1000,

Bu20a, Cell Signalling Technology, Danvers, MA). Following overnight incubation, sections and

coverslips were washed with PBS and incubated with Alexa Fluor goat anti rabbit-488/-568 and goat 
494 Technologies).

Slides were imaged with an AxioObserver inverted microscope (Car Zeiss, Jena, Germany) using 20X Plan-Apochromat/0.80NA or 40X Plan-Apochromat oil/1.4NA objectives (Carl Zeiss). An ApoTome .2 structured illumination device (Carl Zeiss) set at full Z-stack mode and 5 phase images was used for image acquisition. For quantification of BrdU signal in column trophoblasts, 2-4 cell columns per explant ( $\mathrm{n}=9$; obtained using a $20 \mathrm{X}$ objective) were used to calculate values; BrdU+ cell proportions were calculated by BrdU/KRT7+ cells per column; BrdU fluorescence intensity thresholds were used to calculate BrdU proportions within Matrigel explant columns. Images were obtained using an Axiocam 506 monochrome digital camera and processed and analyzed using ZenPro software (Carl 503 Zeiss).

Immunohistochemistry: Decidua (10 weeks' gestation; $\mathrm{n}=1)$ from a first trimester pregnancy was fixed in $2 \%$ paraformaldehyde for $24 \mathrm{hr}$ at $4{ }^{\circ} \mathrm{C}$, paraffin imbedded, and serially sectioned at $6 \mu \mathrm{m}$ onto glass slides. Heat-induced antigen retrieval was performed using sodium citrate (10mM, $\mathrm{pH} 6.0)$ followed by quenching endogenous peroxidases with 3\% hydrogen peroxide for 30 minutes at RT. Sections were then permeabilized with $0.2 \%$ Triton-X-100 for 5 minutes at RT. Serum block was performed with 5\% BSA in tris-buffered saline with $0.05 \%$ Tween 20 (TBST). Sections were then 510 incubated with mouse monoclonal HLA-G (1:100, clone 4H84, ExBio) diluted in TBST overnight at $4^{\circ} \mathrm{C}$. Following overnight incubation, sections were incubated with Envision + Dual Link Mouse/Rabbit 512 HRP-linked secondary antibody (DAKO, Santa Clara, CA) for $1 \mathrm{hr}$ at RT. IgG1 isotype controls and 513 secondary antibody-only negative controls were performed to confirm antibody specificity. Staining 514 was developed via 3,3'-diaminobenzidine (DAB) chromogen (DAB Substrate Kit, Thermo Scientific), 515 counterstained in Modified Harris Hematoxylin Solution (Sigma-Aldrich, St. Louis, MO) and 516 coverslips mounted with Entellan mounting medium (Electron Microscopy Sciences, Hatfield, PA). 
518 (Advanced Cell Diagnostics, Newark, CA) following the manufacturer's instructions (Wang et al., 519 2012). Briefly, placenta $(n=3 ; 6-8$ weeks' gestation) and Matrigel-imbedded placental explants $(n=15$;

5205 explants per oxygen condition) from first trimester pregnancies were fixed overnight at $4{ }^{\circ} \mathrm{C}$ in $4 \%$ 521 paraformaldehyde and paraffin-embedded. Tissue sections were serially sectioned at $6 \mu \mathrm{m}$, and 522 following deparaffinization, antigen retrieval was performed using RNAscope ${ }^{\circledR}$ Target Retrieval 523 Reagent $\left(95^{\circ} \mathrm{C}\right.$ for 15 minutes $)$ and $\mathrm{ACD}$ Protease Plus Reagent $\left(40^{\circ} \mathrm{C}\right.$ for 30 minutes $)$. RNAScope 524 probes targeting LOX (Hs-LOX-C2; Ref. 415941-C2) and negative control (Probe-dapB; Ref. 310043) 525 were incubated on sections for $2 \mathrm{hr}$ at $40^{\circ} \mathrm{C}$, and following this, the RNAscope signal was amplified 526 over 6 rounds of ACD AMP 1-6 incubation and application of RED-A and RED-B at a ratio of 1 527 volume of RED-B to 60 volumes of RED-A for 10 minutes at RT. Selected samples were 528 counterstained with 50\% Hematoxylin I for 2 minutes at RT and all samples were mounted using 529 EcoMount (Biocare Medical, Pacheco, CA).

530

\section{RNA purification}

532

Total RNA was prepared from column and invasive EVT cells using TRIzol reagent (Life 533 Technologies, Carlsbad, CA) followed by RNeasy MinElute Cleanup (Qiagen, Hilden, Germany) and 534 DNase treatment (Life Technologies) according to the manufacturer's instructions. Care was taken so 535 that explants were exposed to atmospheric oxygen no longer than 2 minutes during microscopic 536 dissection and separation of placental villi away from columns prior to the addition of TRIzol. RNA 537 purity was confirmed using a NanoDrop Spectrophotometer (Thermo Fisher Scientific) and by running 538 RNA samples on an Agilent 2100 Bioanalyzer (Agilent Technologies, Santa Clara, CA). Only RNA 539 samples having an RNA Integrity Number (RIN) $>8.0$ were used. 


\section{Microarray hybridization, gene array data preprocessing, gene expression analysis}

544 Centre (McGill University, Montréal, Canada) for RNA quantification. Briefly, RNA samples were 545 prepared for transcriptome profiling using the GeneChip тм Pico Reagent Kit (Thermo Fisher 546 Scientific) as per manufacturer's protocol. Samples were run on the Clariom ${ }^{\text {TM }} \mathrm{S}$ Human Array to 547 measure gene expression at $>20,000$ genes in the human genome (Affymetrix, Santa Clara, CA). Raw 548 data generated from the arrays were read into $\mathrm{R}$ statistical software (version 3.5.1) with the 549 Bioconductor oligo package to convert raw Affymetrix CEL files into an expression matrix of intensity 550 values. The expression data was background corrected, Quantile normalized, and log-transformed. A 551 total of 13,787 control, duplicated, non-annotated or low intensity probes were filtered out of the data, 552 leaving 13,402 probes for further analysis. Pre-processing was monitored at each step by Principal 553 Component Analysis (PCA) and linear modelling. Principal component analysis was performed by the svd ( ) function from the sva package in R. Linear modelling was conducted using the R package

limma. Probe-wise variances were shrunk using empirical Bayes with the eBayes function, followed

genes were defined based on an FDR $<0.05$. Enrichment of pathways were identified and annotated using the clusterProfiler package in Bioconductor (Carvalho and Irizarry, 2010). Volcano plots were generate using the ggplot2 package for RStudio. Cluster analysis of sample relations based on principal components was generated using the plotSampleRelation function for the Lumi package. A hierarchical cluster analysis was conducted using Euclidean distances on the top 40 differentially expressed genes, selected by FDR $<0.05$ and ranked by fold-change $>1.5$.

\section{Single cell RNA-seq data analysis}

565 Processed droplet-based and Smart-seq2 single cell RNA sequencing data was obtained from public 
566 repositories (ArrayExpress experiment codes: E-MTAB-6701 and E-MTAB-6678) (Vento-Tormo et

567 al., 2018). Cells belonging to clusters from trophoblast lineages $(n=14,366)$ were merged from droplet-

568 based and Smart-seq2 data, and corrected for batch-effects using canonical correlation analysis

569 implemented in the R package Seurat (Butler et al., 2018). Pseudotime trajectory modelling was

570 conducted using the monocle $2 \mathrm{R}$ package (Qiu et al., 2017; Trapnell et al., 2014) under the

571 recommended unsupervised procedure called "dpFeature". Briefly, the first 10 principal

572 components on log-normalized expression data were used to construct a TSNE projection, upon which

573 density-peak clustering determined 13 number of clusters, using parameters rho $=2$, delta $=10$. The top

5741000 differentially expressed genes between these clusters were then used for ordering the cells.

575 Visualization of oxygen concentration -dependent genes were visualized along the inferred 576 pesudotime-ordered branches using the $\mathrm{R}$ function

577 monocle::plot_genes_branched_heatmap with the following settings: the number of 578 clusters $\mathrm{k}=5$ for clustering the genes, and default parameters for all else.

\section{LOX activity assay and LOX inhibition}

Measurement of endogenous LOX activity in placental explant conditioned media was

conditioned media from placental explants cultured in triplicate from either $1 \%, 5 \%$, or $20 \%$ oxygen conditions was pooled, concentrated 12-fold using 7.5 kDa exclusion Amicon Millipore concentration

587 the manufacture's instructions (Life Technologies, Carlsbad, CA). This assay is based on the ability of 
590 fluorescence signal measurable at $560 \mathrm{~nm} / 590 \mathrm{~nm}$ excitation/emission wavelengths and was detected 591 using a fluorescence plate reader (BMG Labtech, Ortenberg, Germany) using a 96-well format. As a 592 positive control, $10 \mu \mathrm{g} / \mathrm{ml}$ recombinant active LOX (MyBioscource.com, San Diego, CA) was 593 separately incubated with DAP substrate. LOX activity/specificity was determined by co-incubating 594 reactions with $5 \mathrm{mM}$ of BAPN. For endogenous inhibition of LOX within explant cultures, following $59524 \mathrm{hr}$ of explant establishment at $37{ }^{\circ} \mathrm{C}, 5 \% \mathrm{CO} 2,5 \% \mathrm{O} 2$ in a humidified tri-gas incubator, explant media was replaced with media containing $500 \mu \mathrm{M}$ of BAPN and explant cultures were placed into $1 \%$ 597 or $20 \% \mathrm{O}_{2}$ culture conditions for an additional $48 \mathrm{hr}$ of culture prior to being imaged $/ \mathrm{measured}$.

598

\section{BrdU incorporation assay}

Pulse-chase labeling with BrdU (Sigma-Aldrich) was conducted on a verification cohort of 601 Matrigel embedded placental explants ( $n=3$ distinct placentae, 4-11 columns per oxygen condition). 602 Explants were established in 5\% oxygen for $24 \mathrm{hr}$ followed by $24 \mathrm{hr}$ of culture in either $1 \%, 5 \%$ or $60320 \%$ oxygen. After $48 \mathrm{hr}$ of culture, explants were exposed to a 4-hr pulse with culture media 604 containing $10 \mu \mathrm{M}$ of BrdU. Following $4 \mathrm{hr}$ of labelling, explants were washed in PBS and fixed in $4 \%$ 605 PFA overnight. Explants were paraffin embedded and sectioned for immunofluorescence microscopy. 606 Immunofluorescent staining with anti-BrdU antibody (Bu20a, Sigma-Aldrich) with the addition of a 607 30-minute incubation in 2M hydrochloric acid between permeabilization and sodium borohydride 608 steps.

609

\section{Statistical analysis}

Data are reported as median values with standard deviations. All calculations were carried out

612 using GraphPad Prism software (San Diego, CA). For single comparisons, Mann-Whitney non613 parametric unpaired t-tests were performed. For multiple comparisons, one-way Kruskal-Wallis 
614 ANOVA followed by Dunn's multiple comparison test was performed on explant outgrowth data as 615 outgrowth in 1\% oxygen was not normally distributed. One-way ANOVA followed by Tukey post-test 616 were performed for all other multiple comparisons. The differences were accepted as significant at $P<$ 617 0.05. For gene microarray and scRNA-seq statistical analyses, please refer to the Gene Array Data 618 Preprocessing and scRNA-seq analysis sections in methods.

619

620 


\section{DATA AVAILABILITY/ACCESSION NUMBER}

622 The GEO accession number for the data reported in this paper is: GSE132421

623

624 AUTHOR CONTRIBUTIONS

625 AGB designed the research. AGB, JT, VY, JB, HL, and BC performed experiments and 626 analysed data. AGB, JT, VY, and WPR wrote the paper. All authors read and approved the manuscript.

FUNDING

This work was supported by a Natural Sciences and Engineering Research Council of Canada

(NSERC; RGPIN-2014-04466) Discovery Grant (to AGB), Canadian Institutes of Health Research

632 Master's graduate studentship (to JT).

633

\section{ACKNOWLEDGEMENTS}

The authors extend their sincere gratitude to the hard work of staff at British Columbia's

636 Women's Hospital's CARE Program for recruiting participants to our study, and thank Dr. Megan K.

637 Barker, for her critical readings of the manuscript.

638

639

\section{COMPETING INTERESTS}

640 The authors declare that no competing interests exist. 


\section{REFERENCES}

Abed, M., Verschueren, E., Budayeva, H., Liu, P., Kirkpatrick, D. S., Reja, R., Kummerfeld, S. K., Webster, J. D., Gierke, S., Reichelt, M., et al. (2019). The Gag protein PEG10 binds to RNA and regulates trophoblast stem cell lineage specification. PLOS ONE 14, e0214110.

Aghababaei, M., Hogg, K., Perdu, S., Robinson, W. P. and Beristain, A. G. (2015). ADAM12directed ectodomain shedding of E-cadherin potentiates trophoblast fusion. Cell Death Differ. 22, 1970-1984.

Aghababaei, M., Perdu, S., Irvine, K. and Beristain, A. G. (2014). A disintegrin and metalloproteinase 12 (ADAM12) localizes to invasive trophoblast, promotes cell invasion and directs column outgrowth in early placental development. Mol. Hum. Reprod. 20, 235-249.

Avagliano, L., Marconi, A. M., Romagnoli, S. and Bulfamante, G. P. (2012). Abnormal spiral arteries modification in stillbirths: the role of maternal prepregnancy body mass index. 25, 27892792.

Bang, H., Ha, S. Y., Hwang, S. H. and Park, C.-K. (2015). Expression of PEG10 Is Associated with Poor Survival and Tumor Recurrence in Hepatocellular Carcinoma. Cancer Res Treat 47, 844-852.

Becht, E., McInnes, L., Healy, J., Dutertre, C.-A., Kwok, I. W. H., Ng, L. G., Ginhoux, F. and Newell, E. W. (2018). Dimensionality reduction for visualizing single-cell data using UMAP. Nat. Biotechnol. 37, 38-44.

Benjamini, Y. and Hochberg, Y. (1995). Controlling the False Discovery Rate: A Practical and Powerful Approach to Multiple Testing. Journal of the Royal Statistical Society: Series B (Methodological) 57, 289-300.

Bilban, M., Haslinger, P., Prast, J., Klinglmüller, F., Woelfel, T., Haider, S., Sachs, A., Otterbein, L. E., Desoye, G., Hiden, U., et al. (2009). Identification of novel trophoblast invasion-related genes: heme oxygenase-1 controls motility via peroxisome proliferator-activated receptor gamma. Endocrinology 150, 1000-1013.

Butler, A., Hoffman, P., Smibert, P., Papalexi, E. and Satija, R. (2018). Integrating single-cell transcriptomic data across different conditions, technologies, and species. Nat. Biotechnol. 36, 411-420.

Caniggia, I., Mostachfi, H., Winter, J., Gassmann, M., Lye, S. J., Kuliszewski, M. and Post, M. (2000). Hypoxia-inducible factor-1 mediates the biological effects of oxygen on human trophoblast differentiation through TGFbeta(3). J. Clin. Invest. 105, 577-587.

Carvalho, B. S. and Irizarry, R. A. (2010). A framework for oligonucleotide microarray preprocessing. Bioinformatics 26, 2363-2367.

Chakraborty, D., Cui, W., Rosario, G. X., Scott, R. L., Dhakal, P., Renaud, S. J., Tachibana, M., Rumi, M. A. K., Mason, C. W., Krieg, A. J., et al. (2016). HIF-KDM3A-MMP12 regulatory circuit ensures trophoblast plasticity and placental adaptations to hypoxia. Proc. Natl. Acad. Sci. U.S.A. 113, E7212-E7221. 
Chakraborty, D., Rumi, M. A. K., Konno, T. and Soares, M. J. (2011). Natural killer cells direct hemochorial placentation by regulating hypoxia-inducible factor dependent trophoblast lineage decisions. Proc. Natl. Acad. Sci. U.S.A. 108, 16295-16300.

Cowden Dahl, K. D., Fryer, B. H., Mack, F. A., Compernolle, V., Maltepe, E., Adelman, D. M., Carmeliet, P. and Simon, M. C. (2005). Hypoxia-inducible factors 1alpha and 2alpha regulate trophoblast differentiation. Molecular and Cellular Biology 25, 10479-10491.

Davies, J. E., Pollheimer, J., Yong, H. E. J., Kokkinos, M. I., Kalionis, B., Murthi, P., Davies, J. E., Yong, H. E. J., Kokkinos, M. I., Kalionis, B., et al. (2016). Epithelial-mesenchymal transition during extravillous trophoblast differentiation. Cell Adhesion \& Migration 10, 310-321.

De Luca, L. C., Le, H. T., Mara, D. L. and Beristain, A. G. (2017). ADAM28 localizes to HLA$\mathrm{G}(+)$ trophoblasts and promotes column cell outgrowth. Placenta 55, 71-80.

Dória, S., Sousa, M., Fernandes, S., Ramalho, C., Brandão, O., Matias, A., Barros, A. and Carvalho, F. (2010). Gene expression pattern of IGF2, PHLDA2, PEG10 and CDKN1C imprinted genes in spontaneous miscarriages or fetal deaths. Epigenetics 5, 444-450.

Farrell, A., Alahari, S., Ermini, L., Tagliaferro, A., Litvack, M., Post, M. and Caniggia, I. (2019). Faulty oxygen sensing disrupts angiomotin function in trophoblast cell migration and predisposes to preeclampsia. JCI Insight 4.

Fock, V., Plessl, K., Draxler, P., Otti, G. R., Fiala, C., Knöfler, M. and Pollheimer, J. (2015a). Neuregulin-1-mediated ErbB2-ErbB3 signalling protects human trophoblasts against apoptosis to preserve differentiation. J. Cell. Sci. 128, 4306-4316.

Fock, V., Plessl, K., Fuchs, R., Dekan, S., Milla, S. K., Haider, S., Fiala, C., Knöfler, M. and Pollheimer, J. (2015b). Trophoblast subtype-specific EGFR/ERBB4 expression correlates with cell cycle progression and hyperplasia in complete hydatidiform moles. Hum. Reprod. 30, 789799.

Genbacev, O., Zhou, Y., Ludlow, J. W. and Fisher, S. J. (1997). Regulation of human placental development by oxygen tension. Science 277, 1669-1672.

Gultice, A. D., Kulkarni-Datar, K. and Brown, T. L. (2009). Hypoxia-inducible factor 1alpha (HIF1A) mediates distinct steps of rat trophoblast differentiation in gradient oxygen. Biology of Reproduction 80, 184-193.

Haider, S., Meinhardt, G., Saleh, L., Fiala, C., Pollheimer, J. and Knöfler, M. (2016). Notch1 controls development of the extravillous trophoblast lineage in the human placenta. Proc. Natl. Acad. Sci. U.S.A. 113, E7710-E7719.

Haider, S., Meinhardt, G., Saleh, L., Kunihs, V., Gamperl, M., Kaindl, U., Ellinger, A., Burkard, T. R., Fiala, C., Pollheimer, J., et al. (2018). Self-Renewing Trophoblast Organoids Recapitulate the Developmental Program of the Early Human Placenta. Stem Cell Reports 11, 537-551.

Horii, M., Li, Y., Wakeland, A. K., Pizzo, D. P., Nelson, K. K., Sabatini, K., Laurent, L. C., Liu, Y. and Parast, M. M. (2016). Human pluripotent stem cells as a model of trophoblast differentiation in both normal development and disease. Proc. Natl. Acad. Sci. U.S.A. 113, E3882- 
91.

Ishii, S., Yamashita, K., Harada, H., Ushiku, H., Tanaka, T., Nishizawa, N., Yokoi, K., Washio, M., Ema, A., Mieno, H., et al. (2017). The H19-PEG10/IGF2BP3 axis promotes gastric cancer progression in patients with high lymph node ratios. Oncotarget 8, 74567-74581.

James, J. L., Stone, P. R. and Chamley, L. W. (2006). The effects of oxygen concentration and gestational age on extravillous trophoblast outgrowth in a human first trimester villous explant model. Human Reproduction 21, 2699-2705.

Jauniaux, E., Watson, A. and Burton, G. (2001). Evaluation of respiratory gases and acid-base gradients in human fetal fluids and uteroplacental tissue between 7 and 16 weeks' gestation. $Y M O B$ 184, 998-1003.

Kabir-Salmani, M., Shiokawa, S., Akimoto, Y., Sakai, K. and Iwashita, M. (2004). The role of alpha(5)beta(1)-integrin in the IGF-I-induced migration of extravillous trophoblast cells during the process of implantation. Molecular Human Reproduction 10, 91-97.

Koppes, E., Himes, K. P. and Chaillet, J. R. (2015). Partial Loss of Genomic Imprinting Reveals Important Roles for Kcnq1 and Peg10 Imprinted Domains in Placental Development. PLoS ONE 10, e0135202.

Lash, G. E., Otun, H. A., Innes, B. A., Bulmer, J. N., Searle, R. F. and Robson, S. C. (2006). Low oxygen concentrations inhibit trophoblast cell invasion from early gestation placental explants via alterations in levels of the urokinase plasminogen activator system. Biol. Reprod. 74, 403-409.

Lee, C. Q. E., Turco, M. Y., Gardner, L., Simons, B. D., Hemberger, M. and Moffett, A. (2018). Integrin $\alpha 2$ marks a niche of trophoblast progenitor cells in first trimester human placenta. Development 145, dev162305.

Liang, X. Y., Chen, X., Jin, Y. Z., Chen, X. O. and Chen, Q. Z. (2014). Expression and significance of the imprinted gene PEG10 in placenta of patients with preeclampsia. Genet. Mol. Res. 13, $10607-10614$.

Maltepe, E., Krampitz, G. W., Okazaki, K. M., Red-Horse, K., Mak, W., Simon, M. C. and Fisher, S. J. (2005). Hypoxia-inducible factor-dependent histone deacetylase activity determines stem cell fate in the placenta. Development 132, 3393-3403.

Newby, D., Marks, L., Cousins, F., Duffie, E. and Lyall, F. (2005). Villous explant culture: characterization and evaluation of a model to study trophoblast invasion. Hypertens Pregnancy 24, 75-91.

Ono, R., Nakamura, K., Inoue, K., Naruse, M., Usami, T., Wakisaka-Saito, N., Hino, T., SuzukiMigishima, R., Ogonuki, N., Miki, H., et al. (2006). Deletion of Peg10, an imprinted gene acquired from a retrotransposon, causes early embryonic lethality. Nat. Genet. 38, 101-106.

Peng, Y.-P., Zhu, Y., Yin, L.-D., Zhang, J.-J., Wei, J.-S., Liu, X., Liu, X.-C., Gao, W.-T., Jiang, K.-R. and Miao, Y. (2017). PEG10 overexpression induced by E2F-1 promotes cell proliferation, migration, and invasion in pancreatic cancer. J. Exp. Clin. Cancer Res. 36, 30. 
755

756

757

758

759

760

761

762

763

764

765

766

767

768

769

770

771

772

773

774

775

776

777

778

779

780

781

782

783

784

785

786

787

788

789

790

791

Perdu, S., Castellana, B., Yooona, K., Chan, K., DeLuca, L. and Beristain, A. G. (2016). Maternal obesity drives functional alterations in uterine NK cells. JCI Insight $\mathbf{1}$.

Pijnenborg, R., Vercruysse, L. and Carter, A. M. (2011). Deep trophoblast invasion and spiral artery remodelling in the placental bed of the chimpanzee. Placenta 32, 400-408.

Pollheimer, J., Vondra, S., Baltayeva, J., Beristain, A. G. and Knöfler, M. (2018). Regulation of Placental Extravillous Trophoblasts by the Maternal Uterine Environment. Front Immunol 9, 297.

Qiu, X., Mao, Q., Tang, Y., Wang, L., Chawla, R., Pliner, H. A. and Trapnell, C. (2017). Reversed graph embedding resolves complex single-cell trajectories. Nat. Methods 14, 979-982.

Rahat, B., Mahajan, A., Bagga, R., Hamid, A. and Kaur, J. (2017). Epigenetic modifications at DMRs of placental genes are subjected to variations in normal gestation, pathological conditions and folate supplementation. Sci Rep 7, 40774.

Robins, J. C., Heizer, A., Hardiman, A., Hubert, M. and Handwerger, S. (2007). Oxygen tension directs the differentiation pathway of human cytotrophoblast cells. Placenta 28, 1141-1146.

Tilburgs, T., Crespo, Â. C., van der Zwan, A., Rybalov, B., Raj, T., Stranger, B., Gardner, L., Moffett, A. and Strominger, J. L. (2015). Human HLA-G+ extravillous trophoblasts: Immuneactivating cells that interact with decidual leukocytes. Proc. Natl. Acad. Sci. U.S.A. 112, 7219 7224.

Trapnell, C., Cacchiarelli, D., Grimsby, J., Pokharel, P., Li, S., Morse, M., Lennon, N. J., Livak, K. J., Mikkelsen, T. S. and Rinn, J. L. (2014). The dynamics and regulators of cell fate decisions are revealed by pseudotemporal ordering of single cells. Nat. Biotechnol. 32, 381-386.

Turco, M. Y., Gardner, L., Kay, R. G., Hamilton, R. S., Prater, M., Hollinshead, M. S., McWhinnie, A., Esposito, L., Fernando, R., Skelton, H., et al. (2018). Trophoblast organoids as a model for maternal-fetal interactions during human placentation. Nature 213, 1.

Velicky, P., Knöfler, M. and Pollheimer, J. (2016). Function and control of human invasive trophoblast subtypes: Intrinsic vs. maternal control. Cell Adhesion \& Migration 10, 154-162.

Velicky, P., Meinhardt, G., Plessl, K., Vondra, S., Weiss, T., Haslinger, P., Lendl, T., Aumayr, K., Mairhofer, M., Zhu, X., et al. (2018). Genome amplification and cellular senescence are hallmarks of human placenta development. PLoS Genet. 14, e1007698.

Vento-Tormo, R., Efremova, M., Botting, R. A., Turco, M. Y., Vento-Tormo, M., Meyer, K. B., Park, J.-E., Stephenson, E., Polański, K., Goncalves, A., et al. (2018). Single-cell reconstruction of the early maternal-fetal interface in humans. Nature 563, 347-353.

Wakeland, A. K., Soncin, F., Moretto-Zita, M., Chang, C.-W., Horii, M., Pizzo, D., Nelson, K. K., Laurent, L. C. and Parast, M. M. (2017). Hypoxia Directs Human Extravillous Trophoblast Differentiation in a Hypoxia-Inducible Factor-Dependent Manner. The American Journal of Pathology 187, 767-780.

Wallace, A. E., Fraser, R. and Cartwright, J. E. (2012). Extravillous trophoblast and decidual natural killer cells: a remodelling partnership. Hum. Reprod. Update 18, 458-471. 
Wang, F., Flanagan, J., Su, N., Wang, L.-C., Bui, S., Nielson, A., Wu, X., Vo, H.-T., Ma, X.-J. and Luo, Y. (2012). RNAscope: a novel in situ RNA analysis platform for formalin-fixed, paraffinembedded tissues. J Mol Diagn 14, 22-29.

Wiel, C., Augert, A., Vincent, D. F., Gitenay, D., Vindrieux, D., Le Calvé, B., Arfi, V., LalletDaher, H., Reynaud, C., Treilleux, I., et al. (2013). Lysyl oxidase activity regulates oncogenic stress response and tumorigenesis. Cell Death Dis 4, e855-e855.

Xu, X.-H., Jia, Y., Zhou, X., Xie, D., Huang, X., Jia, L., Zhou, Q., Zheng, Q., Zhou, X., Wang, K., et al. (2019). Downregulation of lysyl oxidase and lysyl oxidase-like protein 2 suppressed the migration and invasion of trophoblasts by activating the TGF- $\beta /$ collagen pathway in preeclampsia. Exp. Mol. Med. 51, 20.

\section{Zhou, Y., Fisher, S. J., Janatpour, M., Genbacev, O., Dejana, E., Wheelock, M. and Damsky, C.} H. (1997). Human cytotrophoblasts adopt a vascular phenotype as they differentiate. A strategy for successful endovascular invasion? J. Clin. Invest. 99, 2139-2151. 


\section{TITLES AND LEGENDS TO FIGURES}

808 Figure 1. Establishment of a human placental villous explant model. (A) Schematic illustration 809 showing trophoblast subtypes within placental villus, anchoring column, and maternal uterine tissue. 810 Depicted are villous cytotrophoblasts (CTB), syncytiotrophoblast (SCT), proximal and distal column 811 trophoblast (PCT \& DCT), and invasive interstitial and endovascular EVT. (B) Immunofluorescence 812 and immunohistochemistry images showing Ki67 (red) and HLA-G (green, brown) expression in 813 human first trimester placental (8 weeks' gestation) and decidual (10 weeks' gestation) tissues. Shown 814 are villous cytotrophoblasts (CTB), proximal and distal column trophoblasts (PCT \& DCT), interstitial 815 EVT (iEVT), and the mesenchymal core (MC). Bar $=100 \mu \mathrm{m}$. (C) Images showing gross villous 816 explant establishment and outgrowth as well as localization of KRT7 (magenta; white), HLA-G 817 (green), and Ki67 (magenta) to specific subtypes of trophoblasts within explant columns. Nuclei are 818 shown via DAPI staining (white). Bar $=100 \mu \mathrm{m}$. 
820 Figure 2. Exposure to low oxygen promotes trophoblast column outgrowth. (A) Schematic illustration

821 depicting the experimental approach to establishing and culturing placental explants in $1 \%, 5 \%$, and

$82220 \%$ oxygen. (B) Representative images showing how explant column outgrowth area is measured at 0

$823 \mathrm{hr}$ and $48 \mathrm{hr}$ of culture. The direction of explant outgrowth/invasion is also indicated. (C)

824 Representative images of villous explants ( $n=8$ distinct placentae; multiple explants per placenta)

825 cultured in $1 \%, 5 \%$, and $20 \%$ oxygen. Shown within the inverted (invert) image is the area of column

826 outgrowth at $48 \mathrm{hr}$ of culture. (D) Bar with scatter plots showing the fold-change of column outgrowth

827 between $48 \mathrm{hr}$ and $0 \mathrm{hr}$ of exposure to $1 \%, 5 \%$, or $20 \%$ oxygen. Median values and standard deviations

828 are shown. Statistical analyses between groups were performed using ANOVA and two-tailed Dunn's

829 post-test; significance considered $p<0.05$.

830

831 
832 Figure 3. Comparison of global gene expression patterns between trophoblast columns exposed to $1 \%$,

$8335 \%$, and $20 \%$ oxygen. (A) Schematic illustration highlighting the removal of placental villi and 834 retention of column trophoblasts and invasive EVT for gene expression analysis. (B) Volcano plots 835 showing individual gene-targeting probes differentially expressed between $1 \%$ and $20 \%, 5 \%$ and $20 \%$, 836 and $1 \%$ and $5 \%$ oxygen cultures. X-axis: coefficients from linear model in log2 scale; y axis: negative $837 \log$ base 10 of the false discovery rate (FDR). Black circles indicate probes with an FDR $>0.05$; blue 838 and orange circles indicate under-expressed and over-expressed probes with an FDR $<0.05$. (C) PCA 839 of $1 \%$ (blue), 5\% (purple), and 20\% (red) oxygen cultured explants. (D) Dendrogram depicts 840 hierarchical clustering of $1 \%, 5 \%$, and $20 \%$ oxygen cultured column trophoblasts using Z-scores from 841 the top 40 (DEGS from 1\% vs 20\% comparisons) genes; $\mathrm{n}=5$ per oxygen group. Within the heatmap, 842 brown $=$ low expression; white $=$ mid-level expression; green $=$ high expression. For each sample, 843 oxygen condition $(1 \%=$ blue; $5 \%=$ purple; $20 \%=$ red $)$ is indicated, as is the unique placental 844 sample/patient ID used to generate the explant (indicated by shade of grey). Venn diagram showing the 845 number of shared and unique genes $($ FDR $<0.05)(\mathbf{E})$ up-regulated and $(\mathbf{F})$ down-regulated in $1 \%$ and $8465 \%$ oxygen-cultured explants compared to $20 \%$ oxygen cultures. 
849 Figure 4. Placental explant exposure to atmospheric oxygen promotes column trophoblast 850 proliferation. Gene ontology (GO) pathway analyses of DGE genes indicating the top 10 up-regulated 851 pathways in explants cultures in (A) 1\% oxygen and (B) 20\% oxygen. Adjusted $p$ values (Bonferroni) 852 and the number of genes identified in each pathway category is represented beside each plot. (C) 853 Dendrogram depicts hierarchical clustering of $1 \%, 5 \%$, and $20 \%$ oxygen cultured column trophoblasts 854 using a proliferation-specific GO signature (Nuclear Division; 93 genes). Within the heatmap, brown = 855 low expression; white $=$ mid-level expression; green $=$ high expression. For each sample, oxygen 856 condition $(1 \%=$ blue $5 \%=$ purple; $20 \%=$ red $)$ is indicated, as is the unique placental sample/patient ID 857 used to generate the explant (indicated by shade of grey). (D) Representative immunofluorescence 858 images of BrdU signal (green) within placental explant columns cultured in 1\%, 5\%, or $20 \%$ oxygen. 859 Trophoblasts are identified by KRT7 signal (magenta). Bar $=100 \mu \mathrm{m}$. (E) Bar/scatter plots show 860 quantification of BrdU incorporation into column trophoblasts cultured in $1 \%$, 5\%, or $20 \%$ oxygen. 861 Median values are shown and statistical analyses between groups were performed using ANOVA and 862 two-tailed Tukey post-test; significance $p<0.05$. 
864 Figure 5. Low oxygen drives column cell differentiation along the EVT pathway. (A) Previously 865 published scRNAseq data (Vento-Tormo et al., 2018) for 14,366 trophoblast cells from first trimester 866 placentae $(n=5)$ were selected for investigating the cell-specificity of our list of identified oxygen867 associated gene expression changes. Uniform Manifold Approximation and Projection (UMAP) (Becht 868 et al., 2018) was used to visualize and cluster the cells after subsetting from other cell types in the 869 published dataset. Cells are labelled according to their previous characterization (Vento-Tormo et al., 870 2018). (B) UMAP clusters cells by gene expression of canonical trophoblast marker genes. (C) 871 Heatmap of top 15 genes upregulated in $1 \%$ and $20 \%$ oxygen. Also shown are genes aligning with 872 general trophoblasts, villous cytotrophoblasts (CTB), proximal column trophoblast (PCT), and distal 873 column trophoblast (DCT)/extravillous trophoblast (EVT). Geneset expression patterns are compared 874 to averaged gene expression levels within single-cell informed cell types (CTB, proliferative CTB, 875 EVT, proliferative EVT, SCT). (D) Pseudotime analysis was applied using Monocle 2 (Qiu et al., 2017; 876 Trapnell et al., 2014) to visualize gene expression across trophoblast differentiation. Two lineage 877 trajectories were identified corresponding to the extravillous pathway and villous pathway. A cell state 878 of origin is also shown. (E) The inferred trajectory resulted into two distinct endpoints: one branch 879 leading to cells highly expressing SCT markers (e.g. ERVFRD-1), and another leading to cells highly 880 expressing EVT markers (HLA-G). (F) A heatmap was constructed using inferred pseudotime and the 881 top 15 upregulated genes in $1 \%$ and in $20 \%$ oxygen conditions. Pseudotime was ordered such that the 882 left and right ends represent the EVT and SCT endpoints. Hierarchical clustering was applied to the 883 genes (ordered along the rows) and separated into 5 clusters. 
885 Figure 6. Low oxygen exposure promotes column-specific expression of $L O X$. (A) Gene array box886 plot expression levels of $L O X$ mRNA in placental explants cultured in $1 \%, 5 \%$, and $20 \%$ oxygen. (B) 887 Representative image showing LOX mRNA transcript in situ localization (dark pink signal) within a 888 first trimester placental villus. The hashed box outlines the enlarged region to the right. Shown are 889 annotations of trophoblast/placental subtypes; Mesenchymal core (MC), cytotrophoblast (CTB), 890 syncytiotrophoblast (SCT), proximal column trophoblast (PCT), distal column trophoblast (DCT). 891 Shown as an inset is an immunofluorescent image depicting nuclei (DAPI; blue), keratin-7 (K7; 892 magenta), and HLA-G (orange) localization within a serial section of the same placental villus. Bars = $893100 \mu \mathrm{m}$. (C) Representative images of $L O X$ mRNA localization within placental explants cultured in $8941 \%$ or $20 \%$ oxygen. Specific trophoblast subtypes are indicated as above, and the hashed box 895 corresponds to the enlarged area shown below. Also shown are insets of immunofluorescent images 896 depicting nuclei (DAPI; blue), keratin-7 (K7; magenta), and HLA-G (orange) localization within 897 corresponding serial sectioned regions of the explant. Bars $=100 \mu \mathrm{m}$. (D) Bar/scatter plots show LOX 898 activity levels within conditioned media (CM) of placental explants cultured in either $1 \%$ or $20 \%$ 899 oxygen in the presence/absence of the LOX inhibitor BAPN (5 mM). Recombinant active lysyl oxidase 900 (rLOX) served as a positive control whereas explant culture media alone served as a negative control. 901 Activity corresponds to level of fluorescence intensity (590 nm). Median values are shown and 902 statistical analyses between groups were performed using ANOVA and two-tailed Tukey post-test; 903 significance $p<0.05$. 
906 Figure 7. Inhibition of lysyl oxidase (LOX) dampens column outgrowth. (A) Bar/scatter plots show

907 LOX activity levels within conditioned media (CM) of placental explants cultured in $1 \%$ oxygen in the 908 presence/absence of BAPN $(500 \mu \mathrm{M})$. Recombinant active lysyl oxidase (rLOX) served as a positive 909 with explant culture media alone served as a negative control. Activity corresponds to level of 910 fluorescence intensity $(590 \mathrm{~nm})$. Median values are shown and statistical analyses between groups were 911 performed using ANOVA and two-tailed Tukey post-test. (B) Representative images of villous 912 explants ( $\mathrm{n}=3$ distinct placentae; multiple explants per placenta) cultured in $1 \%$ oxygen and in the 913 presence/absence of BAPN $(500 \mu \mathrm{M})$. Column outgrowth is shown at $0 \mathrm{hr}$ and $48 \mathrm{hr}$ of culture. (C) Box 914 plots showing the fold-change of column outgrowth between $48 \mathrm{hr}$ and $0 \mathrm{hr}$ following treatment with 915 BAPN. Median values are shown and statistical analyses between groups were performed using 916 ANOVA and two-tailed Tukey post-test; significance $p<0.05$. 


\section{Supplemental Figure Legends}

920 Supplemental Figure 1. Normalization and PCA analysis of placental explant gene microarray data.

921 Average expression values for each explant sample (A) before and (B) after quantile normalization and

922 filtering/removal of probes. (C and D) Principal component analysis (PCA) of raw data, normalized 923 data, and filtered data.

924

925 Supplemental Figure 2. Pathway analyses of unique gene signatures of $1 \%$ and 5\% oxygen-cultured 926 explants. (A) Top 20 gene pathways upregulated exclusively in $1 \%$ oxygen when compared to $20 \%$ 927 oxygen. (B) Top 20 gene pathways exclusively upregulated in 5\% oxygen when compared to $20 \%$ 928 oxygen. The size of the dot represents the number of significant genes in the pathway and the colour 929 represents adjusted $P$-values as indicated in the legend.

930

931 Supplemental Figure 3. Top gene pathways identified in explant columns cultured in 5\% oxygen. Top 93220 pathways upregulated in $5 \%$ oxygen when compared to $20 \%$ oxygen. The size of the dot represents 933 the number of significant genes in the pathway and the colour represents adjusted $P$-values as indicated 934 in the legend.

935

936 Supplemental Figure 4. Low oxygen culture promotes trophoblastic and EVT gene signatures in 937 placental explants. (A) Dendrogram depicts hierarchical clustering of $1 \%$, 5\%, and $20 \%$ oxygen 938 cultured column trophoblasts using the respective 14-gene signature. The heatmap displays expression 939 profiles of trophoblast genes identified as being significantly different between $1 \%, 5 \%$, and $20 \%$ 940 cultures. Within the heatmap, brown = low expression; white $=$ mid-level expression; green $=$ high 941 expression. For each sample, oxygen condition $(1 \%=$ blue $5 \%=$ purple; $20 \%=$ red $)$ is indicated, as is 942 the unique placental sample/patient ID used to generate the explant (indicated by shade of grey). 
943 Representative immunofluorescence images showing expression of PEG10 (magenta) within (B) first 944 trimester placental villi and (C) Matrigel-imbedded placental explants cultured in either 1\%, 5\%, or $94520 \%$ oxygen. Trophoblast are identified by keratin-7 staining (KRT7; green) and nuclei are stained 946 with DAPI (white). Shown are specific cell types: Syncytiotrophoblast (SCT), villous cytotrophoblasts 947 (CTB), proximal column trophoblast (PCT), distal column trophoblast (DCT), and the mesenchymal 948 core $(\mathrm{MC})$. Bar $=100 \mu \mathrm{m}$. 
bioRxiv preprint doi: https://doi.org/10.1101/669796; this version posted June 13, 2019. The copyright holder for this preprint (which was not certified by peer review) is the author/funder, who has granted bioRxiv a license to display the preprint in perpetuity. It is made available under aCC-BY-NC-ND 4.0 International license.

\section{Figure 1}

A

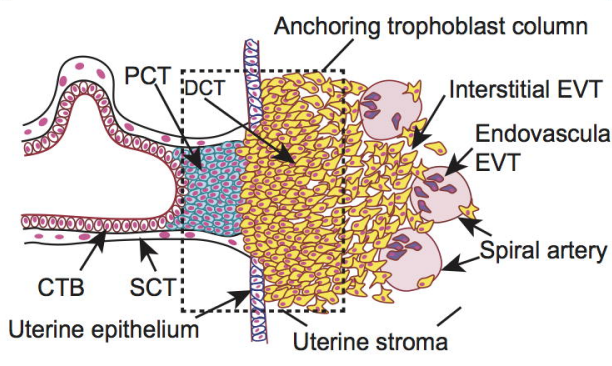

B

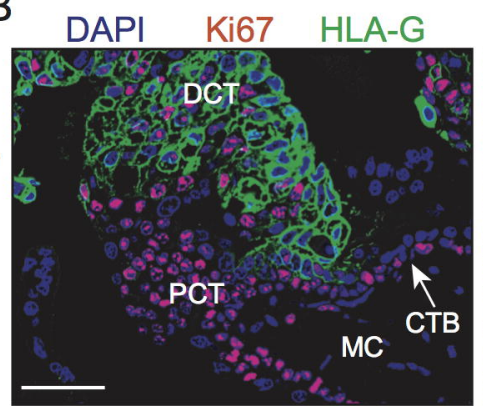

8wk placenta
HLA-G

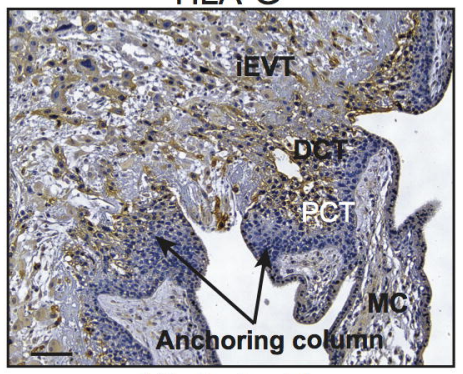

10wk decidua

C
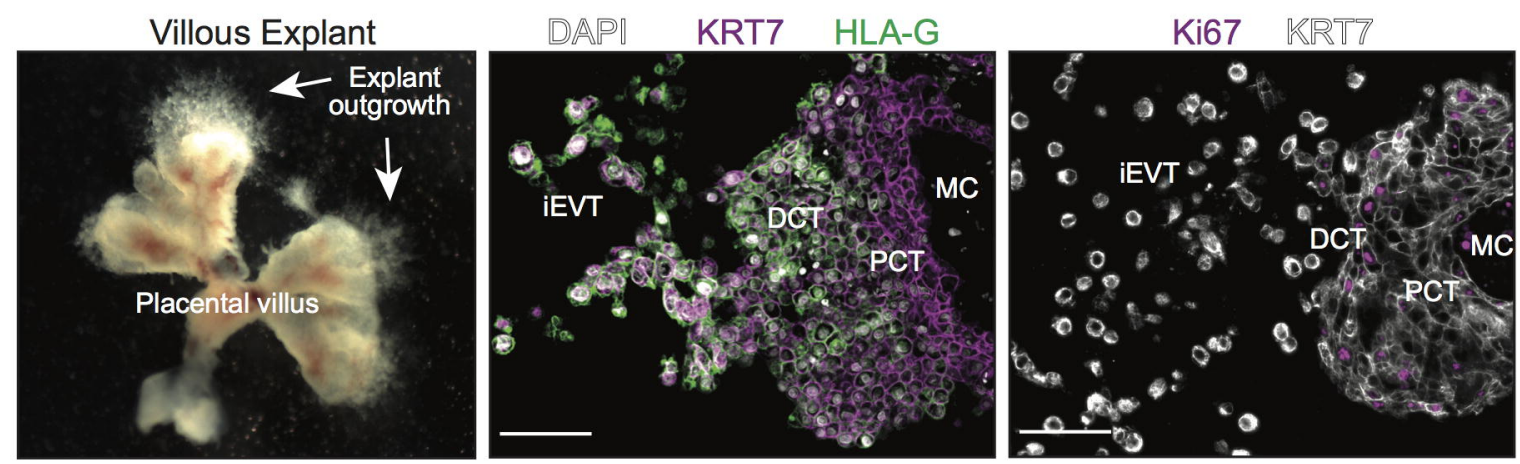
bioRxiv preprint doi: https://doi.org/101101/669796; this version posted June 13,2019 . The copyright holder for this preprint (which was not certified by peer review) is the author/funder, who has granted bioRxiv a license to display the preprint in perpetuity. It is made available under aCC-BY-NC-ND 4.0 International license.

\section{Figure 2}
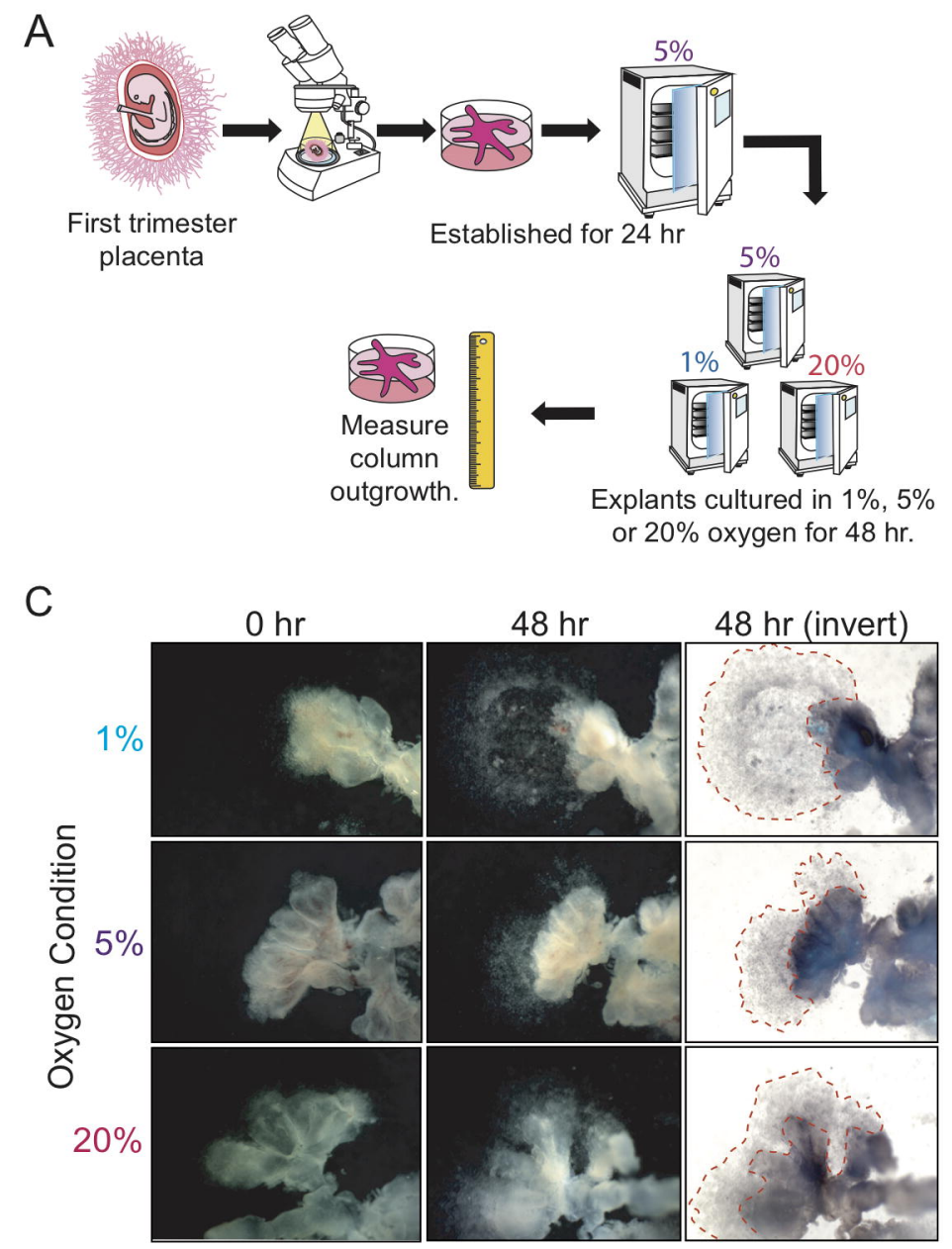

B
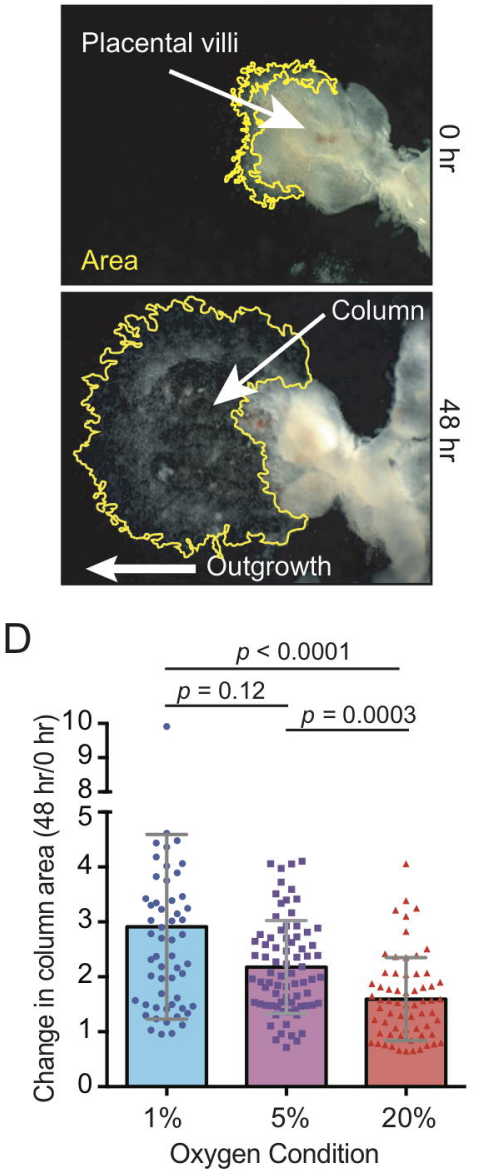
bioRxiv preprint doi: https://doi.org/10.1101/669796; this version posted June 13,2019 . The copyright holder for this preprint (which was not certified by peer review) is the author/funder, who has granted bioRxiv a license to display the preprint in perpetuity. It is made available under aCC-BY-NC-ND 4.0 International license.

\section{Figure 3}

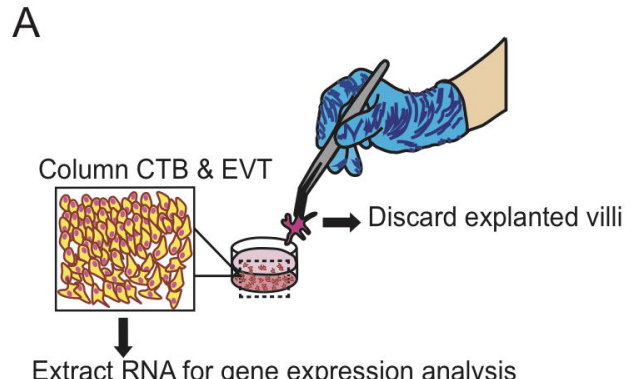

Extract RNA for gene expression analysis

$\mathrm{B}$
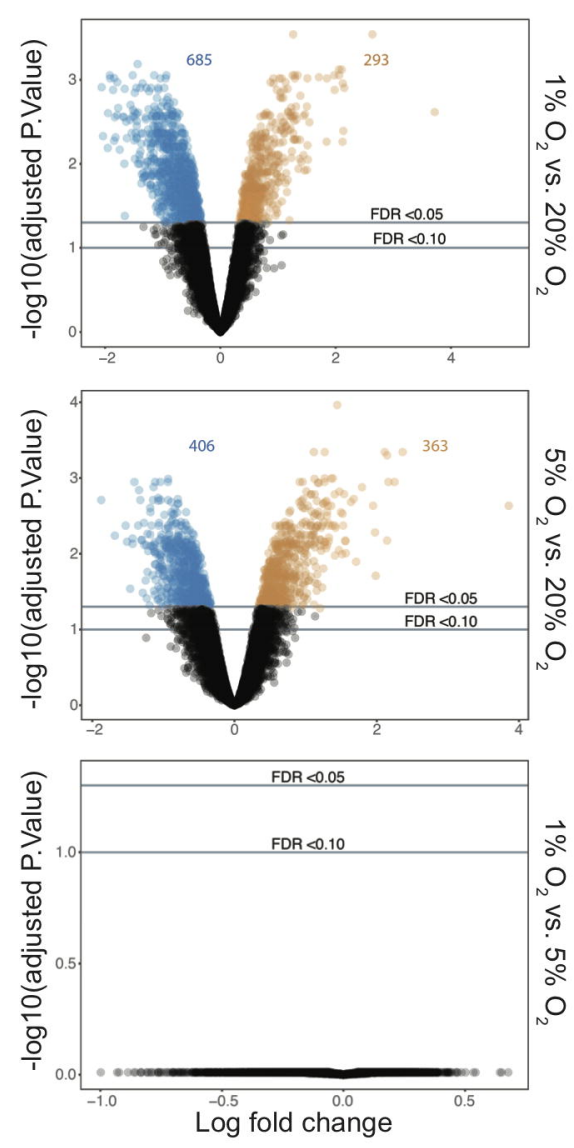

$E$

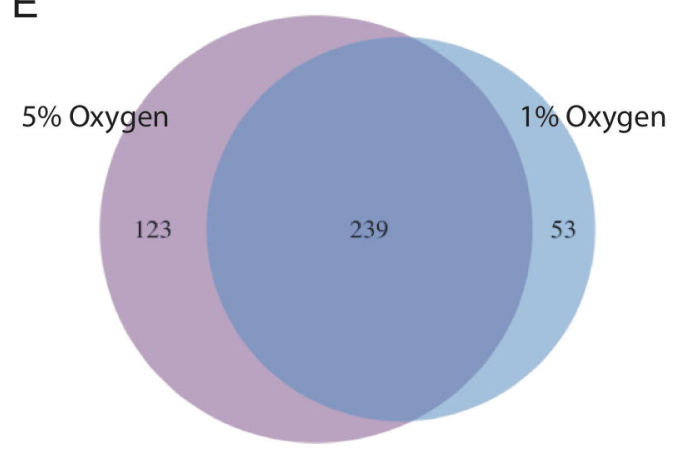

Up-regulated genes
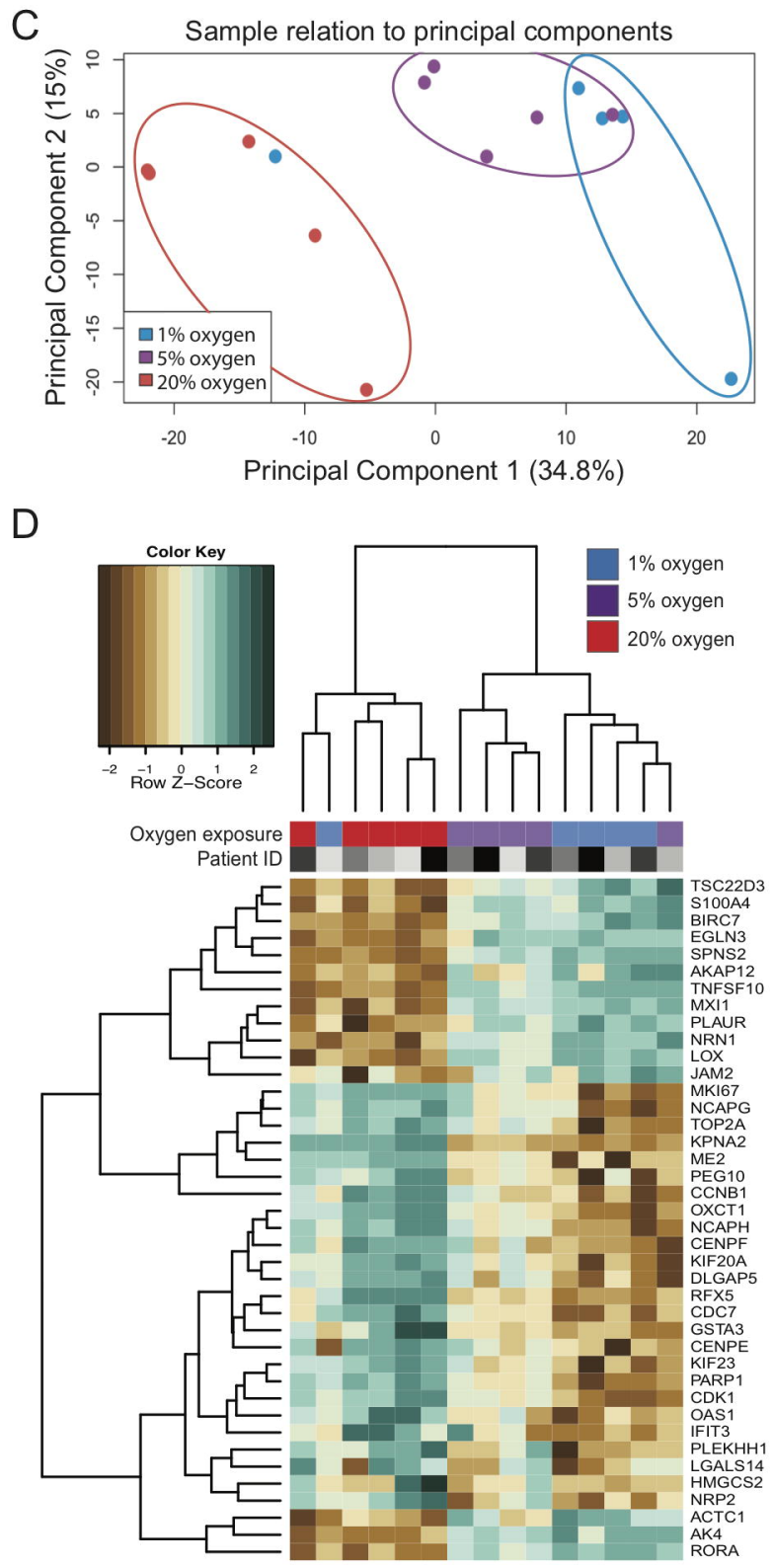

$\mathrm{F}$

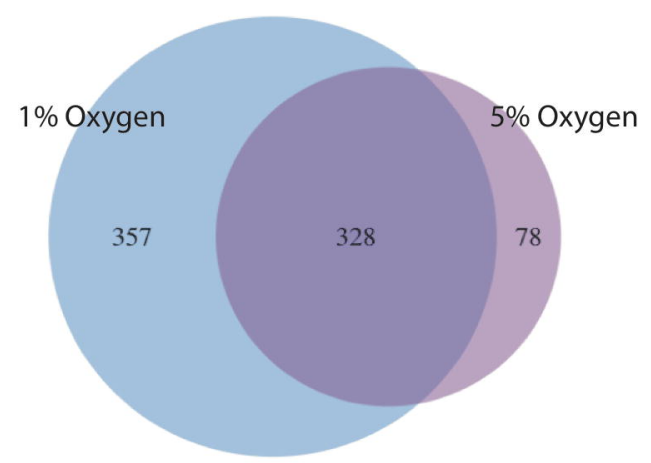

Down-regulated genes 
bioRxiv preprint doi: https://doi.org/10.1101/669796; this version posted June 13,2019. The copyright holder for this preprint (which was not certified by peer review) is the author/funder, who has granted bioRxiv a license to display the preprint in perpetuity. It is made available under aCC-BY-NC-ND 4.0 International license.

\section{Figure 4}

A

C
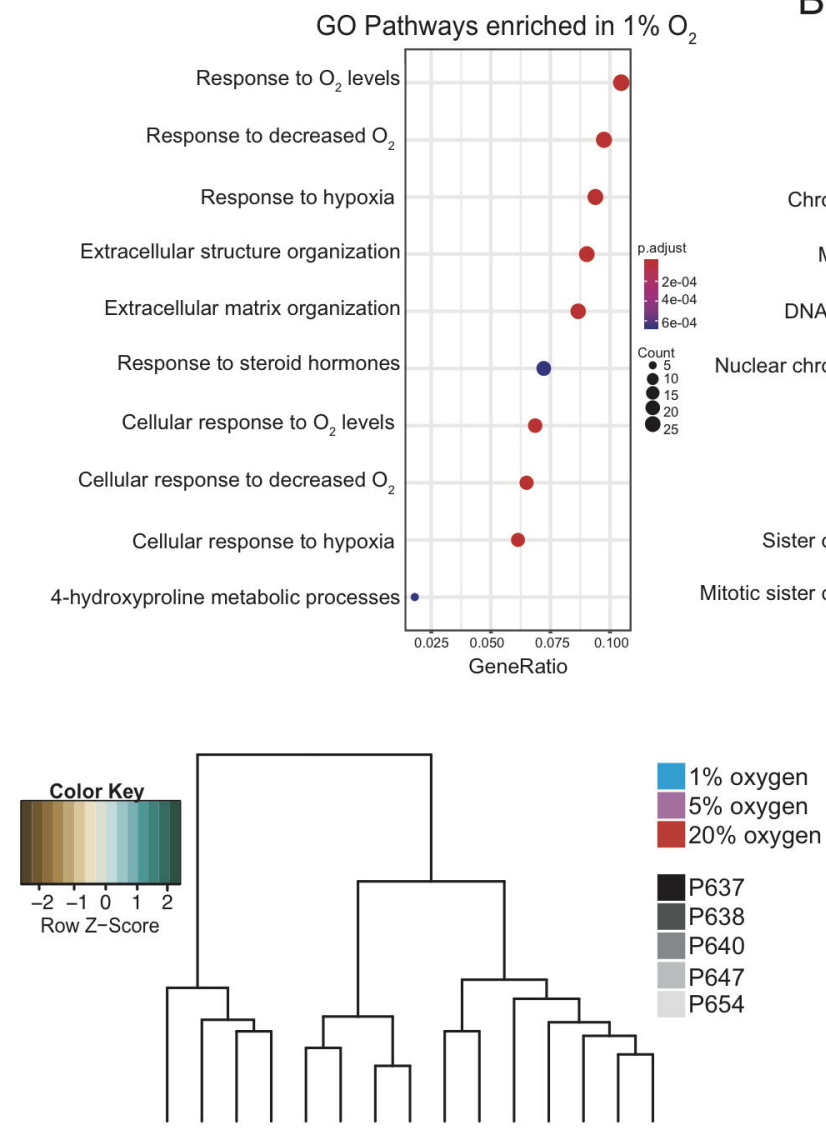

Oxygen Exposure

Patient ID

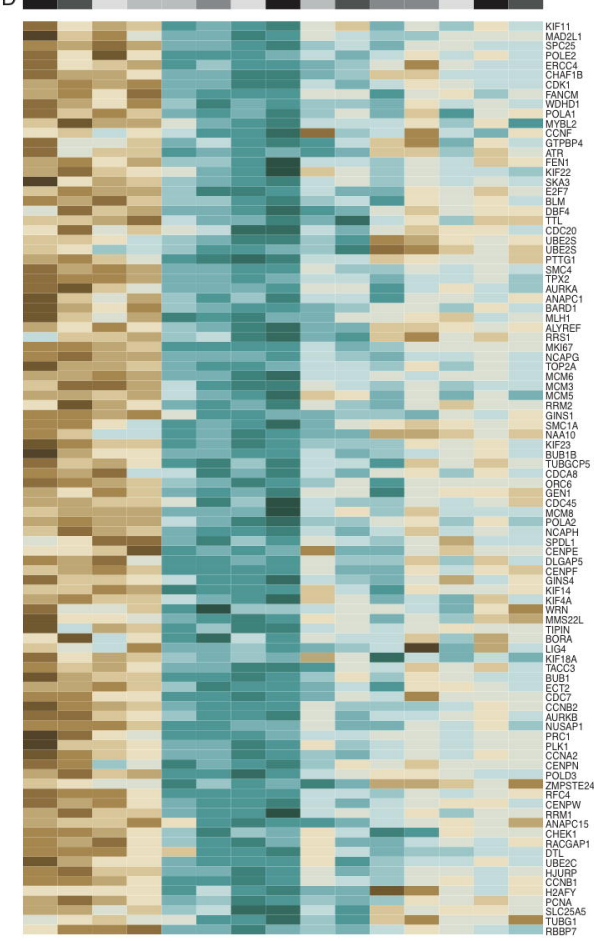

Proliferation gene signature
B

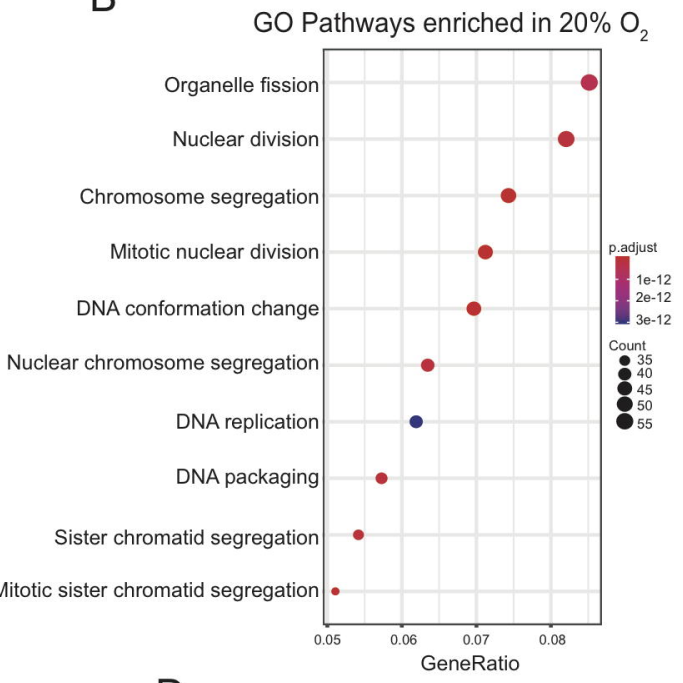

$\mathrm{D}$

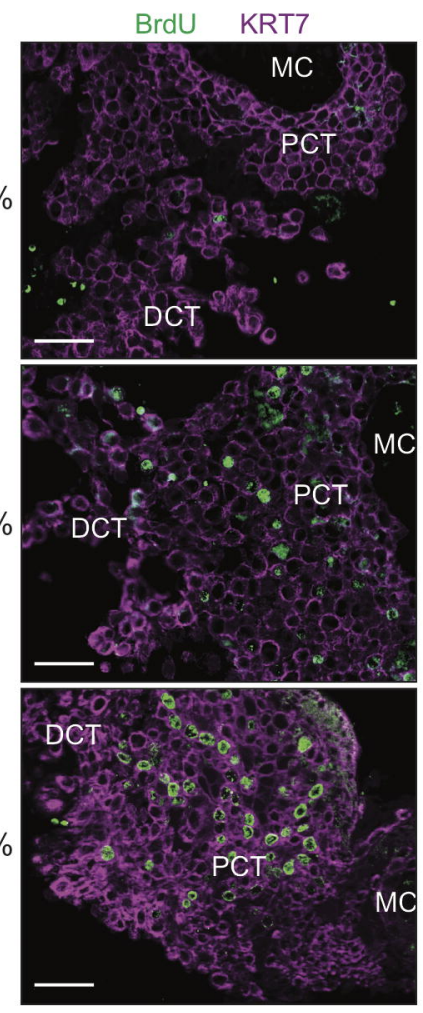

E

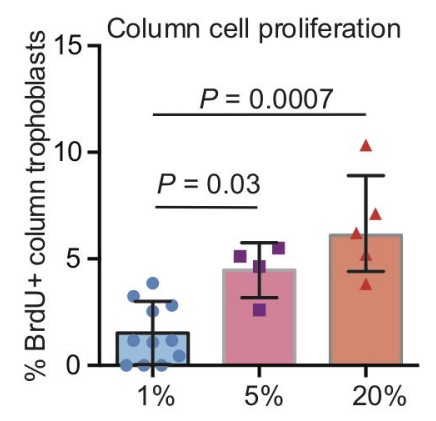


bioRxiv preprint doi: https://doi.org/10.1101/669796; this version posted June 13, 2019. The copyright holder for this preprint (which was not certified by peer review) is the author/funder, who has granted bioRxiv a license to display the preprint in perpetuity. It is made available under aCC-BY-NC-ND 4.0 International license.

\section{Figure 5}

A

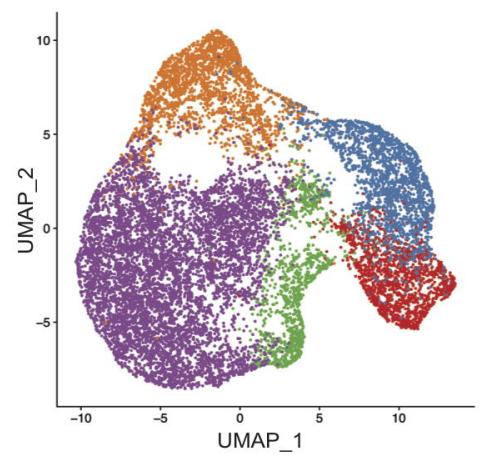

D

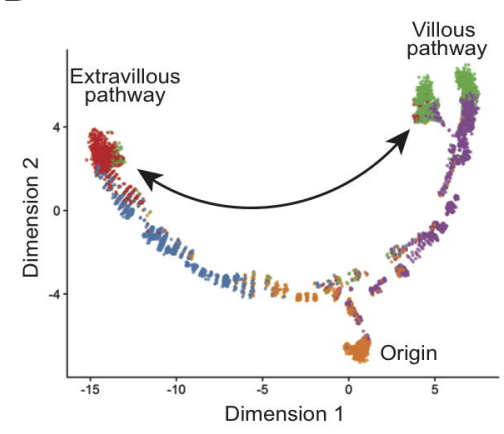

F

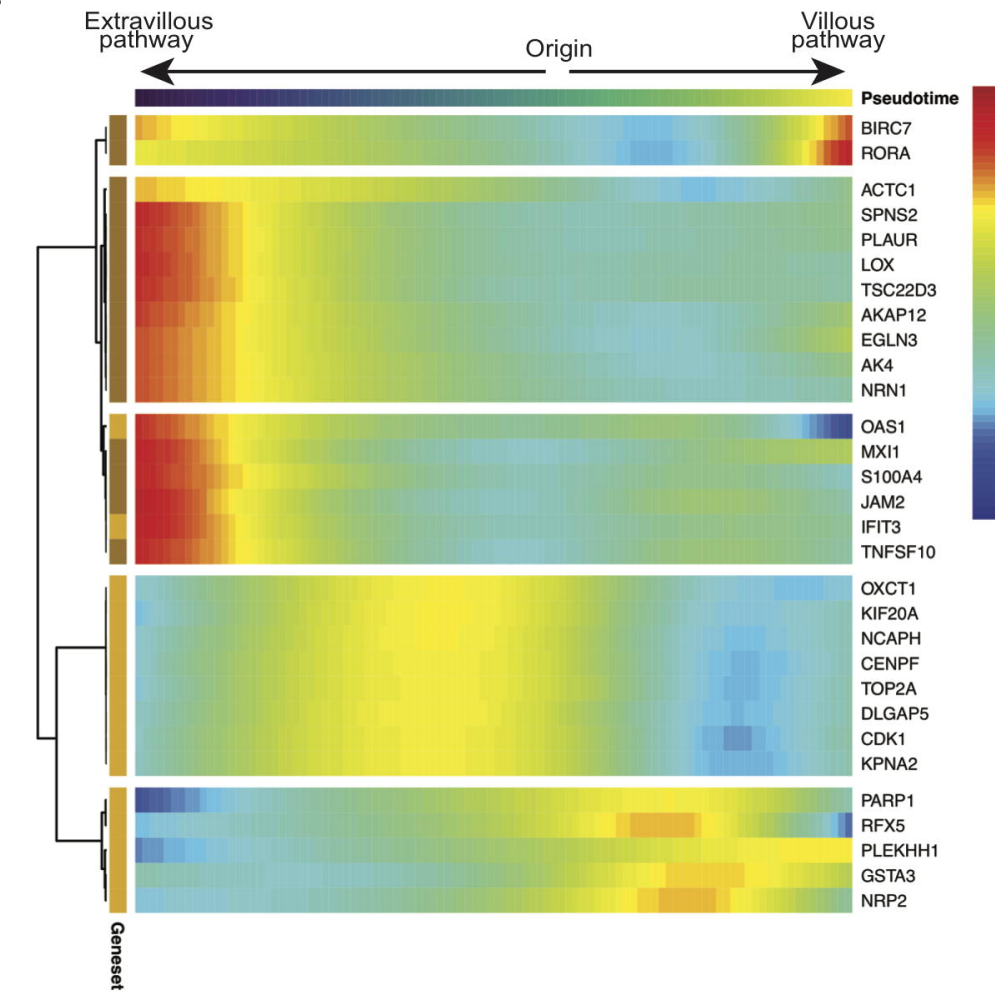

B
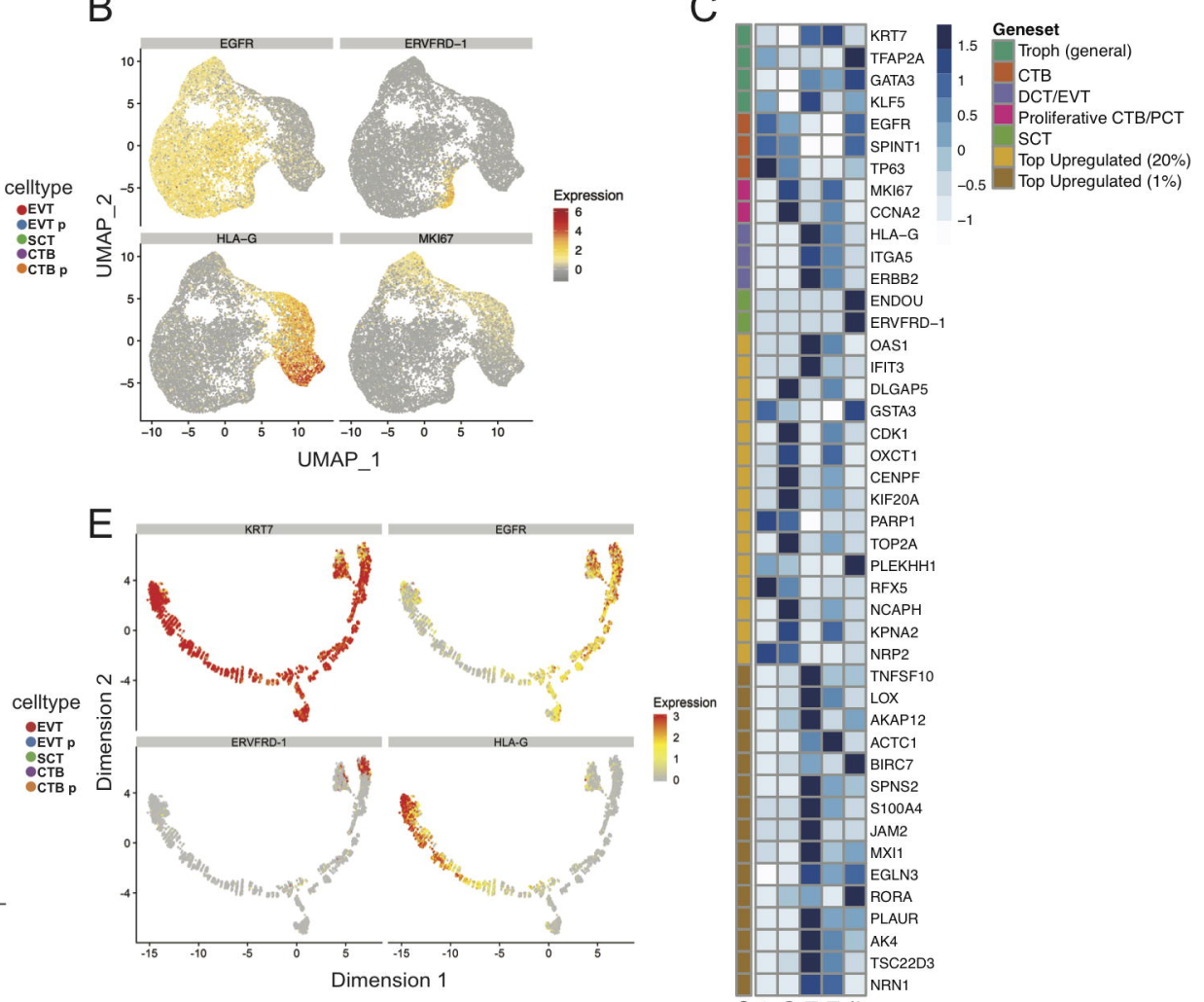
bioRxiv preprint doi: https://doi.org/10.1101/669796; this version posted June 13,2019 . The copyright holder for this preprint (which was not certified by peer review) is the author/funder, who has granted bioRxiv a license to display the preprint in perpetuity. It is made available under aCC-BY-NC-ND 4.0 International license.

\section{Figure 6}
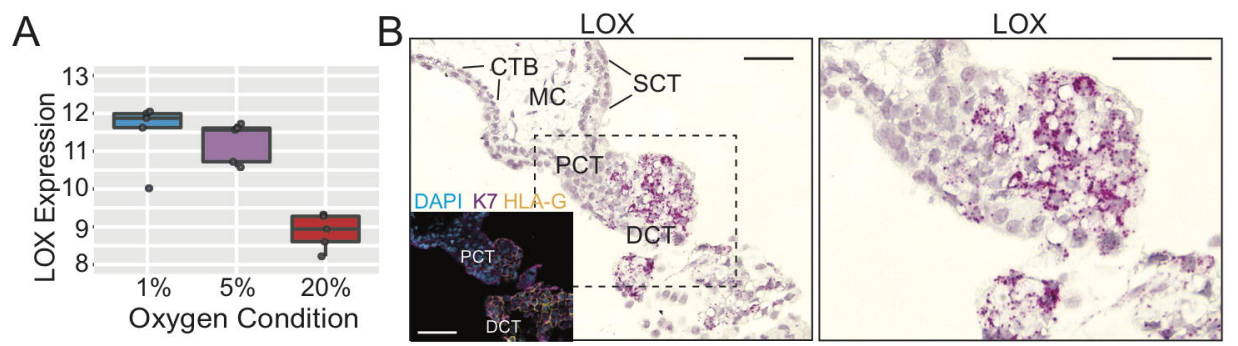

C
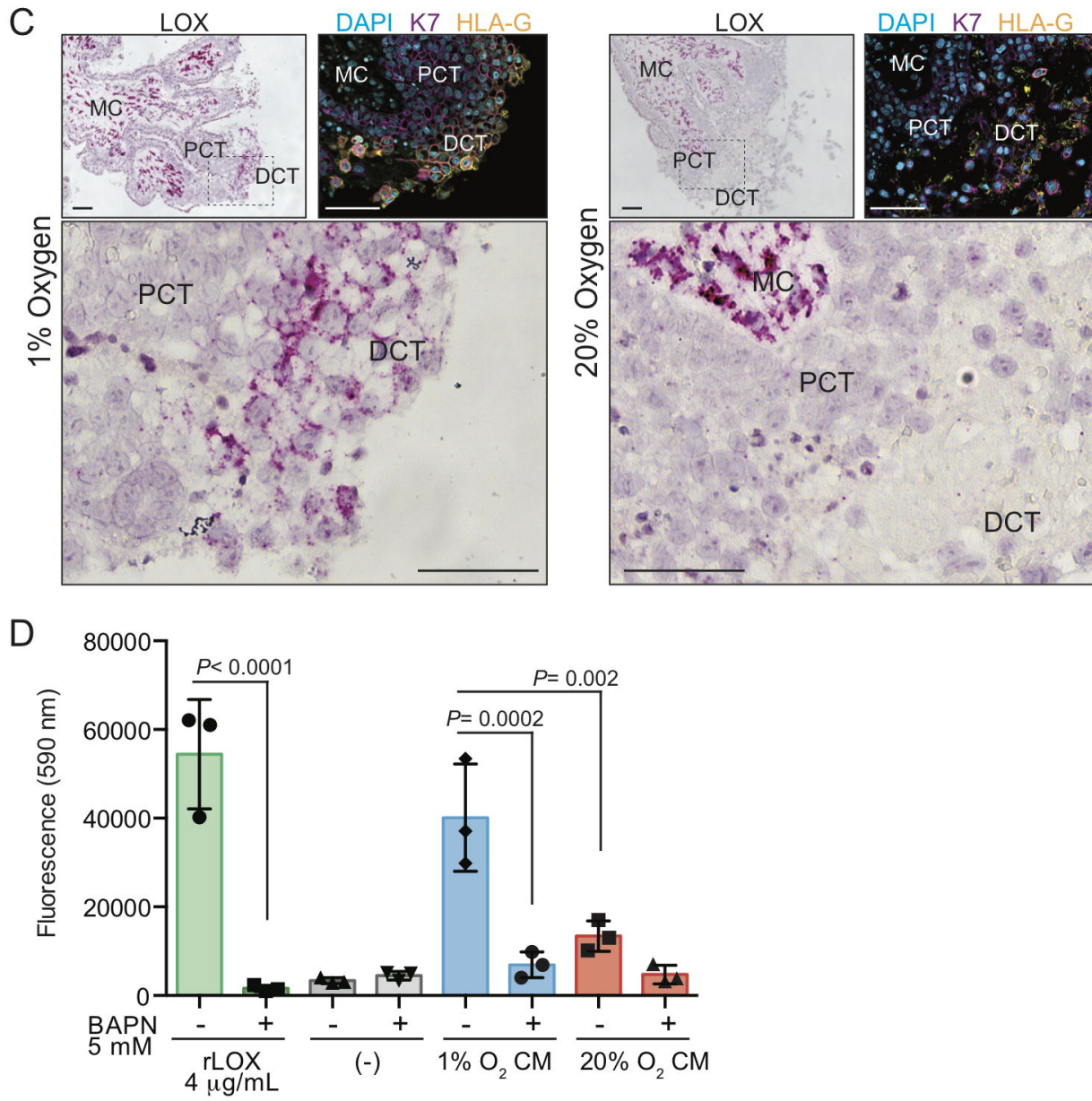
bioRxiv preprint doi: https://doi.org/10.1101/669796; this version posted June 13, 2019. The copyright holder for this preprint (which was not certified by peer review) is the author/funder, who has granted bioRxiv a license to display the preprint in perpetuity. It is made available under aCC-BY-NC-ND 4.0 International license.

\section{Figure 7}
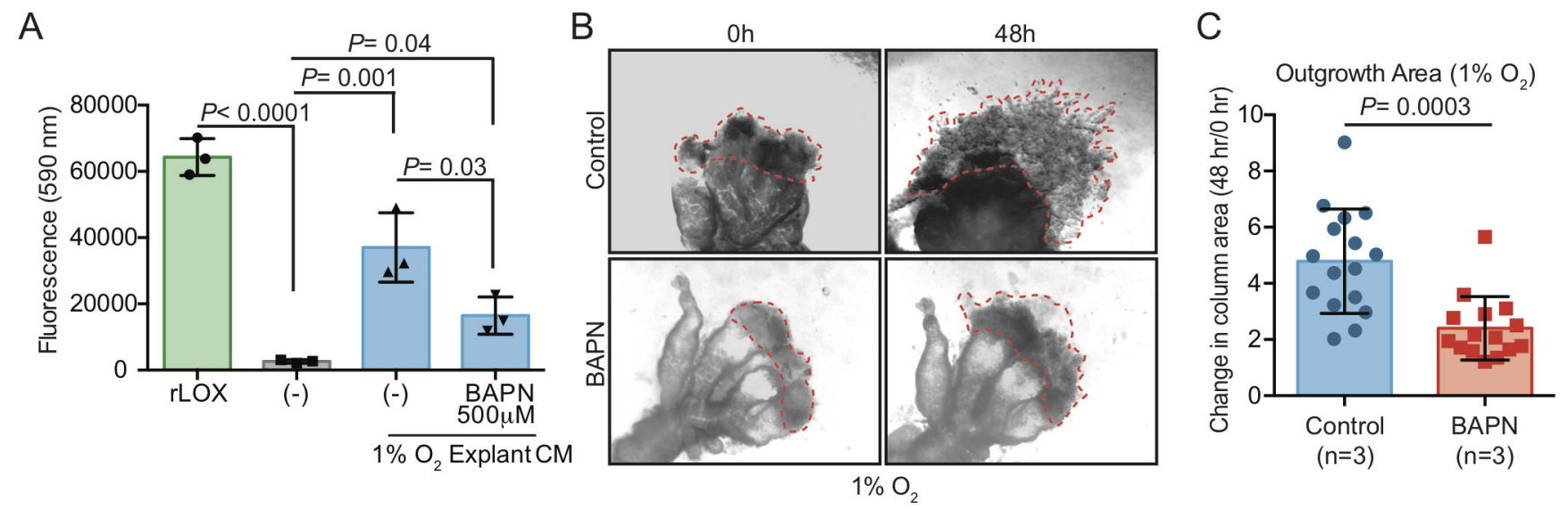